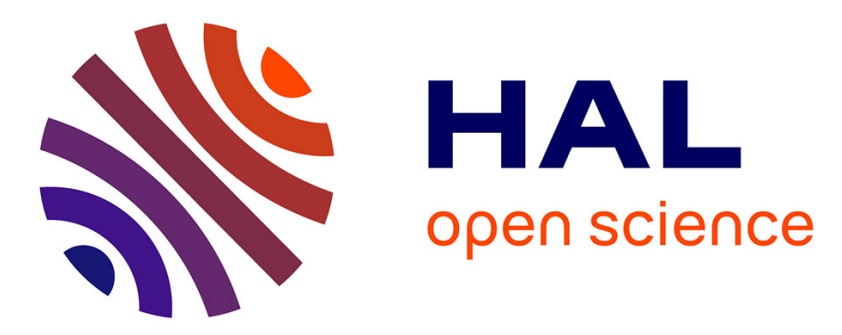

\title{
Nanoforce estimation based on Kalman filtering and applied to a force sensor using diamagnetic levitation
} Emmanuel Piat, Joël Abadie, Stéphane Oster

\section{To cite this version:}

Emmanuel Piat, Joël Abadie, Stéphane Oster. Nanoforce estimation based on Kalman filtering and applied to a force sensor using diamagnetic levitation. Sensors and Actuators A: Physical , 2012, 179, pp.223-236. 10.1016/j.sna.2012.02.025 . hal-01303503

\section{HAL Id: hal-01303503 https://hal.science/hal-01303503}

Submitted on 18 Apr 2016

HAL is a multi-disciplinary open access archive for the deposit and dissemination of scientific research documents, whether they are published or not. The documents may come from teaching and research institutions in France or abroad, or from public or private research centers.
L'archive ouverte pluridisciplinaire HAL, est destinée au dépôt et à la diffusion de documents scientifiques de niveau recherche, publiés ou non, émanant des établissements d'enseignement et de recherche français ou étrangers, des laboratoires publics ou privés. 


\title{
Nanoforce estimation based on Kalman filtering and applied to a force sensor using diamagnetic levitation
}

\author{
Emmanuel Piat ${ }^{\mathrm{a}, * *}$, Joël Abadie ${ }^{\mathrm{a}, *}$, Stéphane Oster ${ }^{\mathrm{a}, \mathrm{b}}$ \\ ${ }^{a}$ femto-st Institute, UMR CNRS 6174, UFC - ENSMM - UTBM, Besançon, France \\ ${ }^{b}$ STIL SA, Aix-en-Provence, France
}

\begin{abstract}
Nanoforce sensors based on passive diamagnetic levitation with a macroscopic seismic mass are a possible alternative to classical Atomic Force Microscopes when the force bandwidth to be measured is limited to a few Hertz. When an external unknown force is applied to the levitating seismic mass, this one acts as a transducer that converts this unknown input into a displacement that is the measured output signal. Because the under-damped and long transient response of this kind of macroscopic transducer can not be neglected for time-varying force measurement, it is then necessary to deconvolve the output to correctly estimate the unknown input force. The deconvolution approach proposed in this paper is based on a Kalman filter that use an uncertain a priori model to represent the unknown nanoforce to be estimated. The main advantage of this approach is that the end-user can directly control the unavoidable trade-off that exists between the wished resolution on the estimated force and the response time of the estimation.
\end{abstract}

Keywords: micro and nano force sensor, magnetic spring, Kalman filtering, unknown input estimation

\section{Introduction}

The design of micro and nanoforce sensors is constrained by the fact that only force effects can be directly observed. Because of this, a transducer is necessary to convert the force into a measurable effect. The force is the unknown input to reconstruct and the effect is the measured output signal. Most of the time, the measured force effect is related to a displacement $x$ and the usual scalar expression used to calculate the component $F$ of the applied force $\vec{F}$ in one-direction $\vec{x}$ of space simply consists in the equation:

$$
F=K x \quad K>0
$$

in which $K$ is the mechanical stiffness of the transducer along $\vec{x}$ (by convention $x$ is set to zero when there is no displacement). This steady-state equation supposes that the transient response of the transducer can be neglected. This is usually considered to be the case for classical designs using monolithic elastic microstructures like microcantilevers which have a high resonant frequency [1]: AFM based microforce sensors [2] [3], piezoresistive microforce sensors [4], capacitive microforce sensors [5], piezoelectric microforce sensors [6], etc. When the transient dynamic of the transducer due to the evolution of the successive derivatives of $x$ is not negligible, Eq. (1) can not be used.

\footnotetext{
*Principal corresponding author.

** Corresponding author.

Email addresses: emmanuel.piat@ens2m.fr (Emmanuel Piat), joel.abadie@femto-st.fr (Joël Abadie), contact@stilsa.com (Stéphane Oster)
}

The general framework of the force estimation (shown in Fig. 1) is then equivalent to the deconvolution problematic of a noisy output signal or the design of a unknown input observer. In the specific case treated in this paper, the unknown input is a nanoforce that is applied to a macroscopic seismic mass that levitates passively thanks to the diamagnetic levitation principle. This seismic mass acts as a transducer that converts the unknown input force into a displacement that is the measured output signal corrupted by noise. This kind of macroscopic transducer has a under-damped and long transient response, thus this dynamic behaviour must be taken into account during the estimation process contrary to what is done in Eq. (1). The estimation computation is based on a discrete Kalman filter that use an uncertain a priori model to represent the unknown force to be estimated. This paper begins by a short description of the force sensor and its dynamic behaviour (state-space modelling). The calibration process is then briefly presented and followed by the development of the unknown input estimation under Gaussian assumptions usually used to derive a Kalman filter. To be realistic, some performances of the force estimation obtained are then characterised in a non Gaussian framework and an analysis of the trade-off between resolution and bandwidth is developed using a steady-state Kalman filter. Finally, some experimental results are presented. A glossary of all mathematic symbols used is also present at the end of the article. 


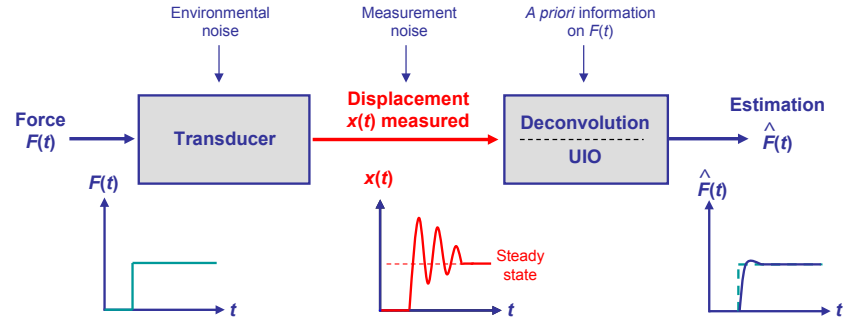

Figure 1: Force estimation using a deconvolution approach or an unknown input observer (UIO).

\section{Passive micro and nanoforce sensor prototype based on diamagnetic levitation}

\subsection{Sensor description}

Microforce sensors based on "heavy" rigid seismic mass are really uncommon because of the inertia of their mass that should be a great handicap for time-varying force measurement. A force sensor with a range measurement of several millinewtons and based on a mass moving inside a pneumatic linear bearing is described in [7]. The mass is $21.17 \mathrm{~g}$ and the force resolution is 0.5 micronewton. The air friction inside the bearing is assumed small enough to be neglected. A force sensor based on magnetic springs and upthrust buoyancy is presented in $[8,9]$. The seismic mass is a float. Its weight is 4 grams. Resolution is around $10 \mathrm{nN}$ and measurement range is $\pm 50 \mu \mathrm{N}$. This force sensor can measure the horizontal component of an external force and the vertical component of an external torque. The design presented in this paper is also based on magnetic springs like [8] but uses repulsive diamagnetic force $[10,11]$ instead of repulsive upthrust buoyancy to stabilise the seismic mass. It reaches the same force resolution than an Atomic Force Microscope (AFM) but with a larger range measurement. This design is based on a macroscopic mass $(\approx 70 \mathrm{mg}$ ) that is levitating passively thanks to to the diamagnetic levitation principle. This mass is a rigid 10-cm long capillary tube made of glass on which are stuck two small magnets $M_{2}$. The whole structure is called maglevtube (see Fig. 2). As it is shown in Fig. 3, the maglevtube levitates passively around a given equilibrium state because of repulsive diamagnetic effects (generated by four graphite diamagnetic plates) coupled with attractive magnetic effects (generated by magnets $M_{1}$ and $M_{1}^{\prime}$ ). The maglevtube has a microscopic tip on which is applied the unknown external force $\vec{F}$. The sensor is currently designed to only measure forces applied along the longitudinal axis $\vec{x}$ of the tube. Thus, the unknown force $\vec{F}$ is assumed to be collinear to $\vec{x}$ and has the following components in the global reference frame $R_{0}$ given in Fig. 3:

$$
\vec{F}\left[\begin{array}{lll}
F^{x} & 0 & 0
\end{array}\right]^{\top}
$$

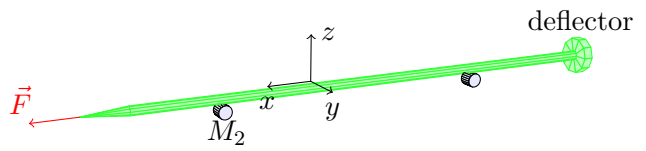

Figure 2: Macroscopic seismic mass sensitive to external forces.

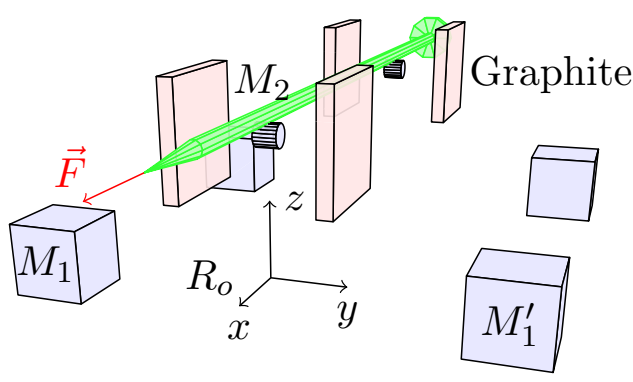

Figure 3: Levitating seismic mass in the force sensing device.

\subsection{Force sensing principle}

The forces involved in the maglevtube dynamic along axis $\vec{x}$ are the external force $\vec{F}$, the viscous friction due to the air and the magnetic force. The maglevtube weight and the diamagnetic force are not involved because they act along axis $\vec{z}$ and $\vec{y}$ respectively. When the force $F^{x}$ is applied to the tube tip, the displacement obtained corresponds to an under-damped behaviour because the viscous friction due to the air is very small. The simulated displacement computed with Matlab-Simulink and obtained with a step force (which amplitude $F^{x}$ is set to $1 \mu \mathrm{N}$ ) is given in Fig. 4 (step response). This simulation of the prototype presented in Section 2.3 is done with a complete computation of the internal magnetic and diamagnetic forces at each time step of the Simulink solver with an non-analytical physical model. Thus, the complete behaviour of the six DOF of the maglevtube can be plotted if necessary. In the case studied here, there is only a dynamic along the axis $\vec{x}$ due to the assumption done in Eq. (2). The settling time at $5 \%$ along $\vec{x}$ axis is typically 20 seconds. Overshoot is $97 \%$. The nonlinear steady-state response of the maglevtube is given in Fig. 5. The slope of this curve corresponds to the magnetic stiffness $K_{m}^{x}$ of the sensor that is equivalent to an invisible magnetic spring with a very small damping. One can notice that the linearity of the stiffness is good with displacements between \pm 1.5 millimetres. For such range of displacements, the maximum relative error between the linearised force and the nonlinear magnetic force is $0.63 \%$ in this simulation [12]. As a consequence, the behaviour of the maglevtube will be assumed linear for displacements between \pm 1.5 millimetres. Knowing the magnetic stiffness $K_{m}^{x}$, the force measurement is given by Eq. (1) in steady-state:

$$
F^{x}=K_{m}^{x} x \quad K_{m}^{x}>0 .
$$

Because the stiffness is equal to $0.0289 \mathrm{~N} / \mathrm{m}$ in this simulation, the corresponding measured force range associated to a \pm 1.5 millimetres range displacement is $\pm 43 \mu \mathrm{N}$. 


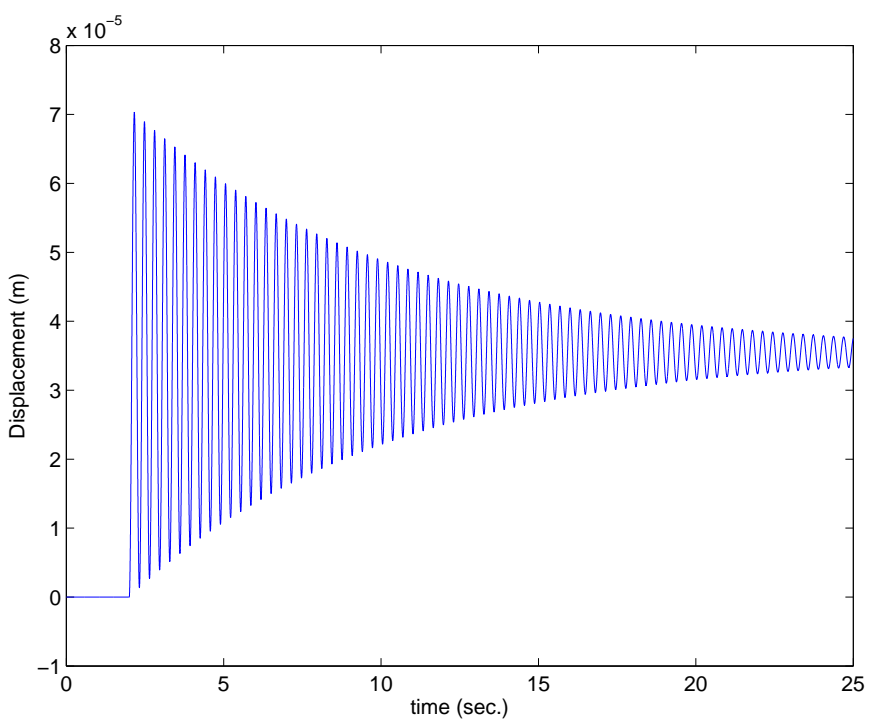

Figure 4: Simulated step response of the maglevtube with an external input force set to one micro newton.

Eq. (3) can not be used for time-varying force measurement, especially if the seismic mass inertia is high. In this case, the dynamic response of the seismic mass must be taken into account in order to correctly estimate the unknown force applied on the mass. This specific problem is addressed in this paper. It is an extended analysis of the study briefly presented in [13] and it completes [12] in which only steady-state measurements are developed using Eq. (3).

\subsection{Experimental prototype}

Typical $K_{m}^{x}$ stiffnesses obtained with the prototype shown in Fig. 6 are between $0.005 \mathrm{~N} / \mathrm{m}$ and $0.03 \mathrm{~N} / \mathrm{m}$ (same order of magnitude than for very flexible AFM cantilevers). The stiffness and the horizontal attitude of the maglevtube can be adjusted by changing the distances between magnets $M_{1}$ and $M_{1}^{\prime}$. Lower is the stiffness and better is the sensitivity of the sensor. There is nevertheless a limitation on the lower value that can be reached for $K_{m}^{x}$ because if magnets $M_{1}$ and $M_{1}^{\prime}$ are too far away from each other, the magnetic force along the vertical axis is not sufficient to compensate the maglevtube weight. Typical mass $m$ for the maglevtube is around $70 \mathrm{mg}$. The sensor used to measure the maglevtube displacement is a confocal chromatic sensor (manufactured by STIL SA) that is aimed at a glass deflector stuck at the rear of the maglevtube (see Fig. 2). The force measurement range and the resolution obtained depends on the measurement range of the confocal head used. For instance, a CL4 (resp. CL2) head haves a $\pm 1.5 \mathrm{~mm}$ (resp. $\pm 150 \mu \mathrm{m}$ ) measurement range and covers the linear domain of the sensor. The associated typical standard deviation of a CL4 (resp. CL2) head is $75 \mathrm{~nm}$ (resp. $12 \mathrm{~nm}$ ). Thus, without any signal processing and in steady-state with $K_{m}^{x}$ set to $0.01 \mathrm{~N} / \mathrm{m}$, the minimal theoretical standard deviation that can be

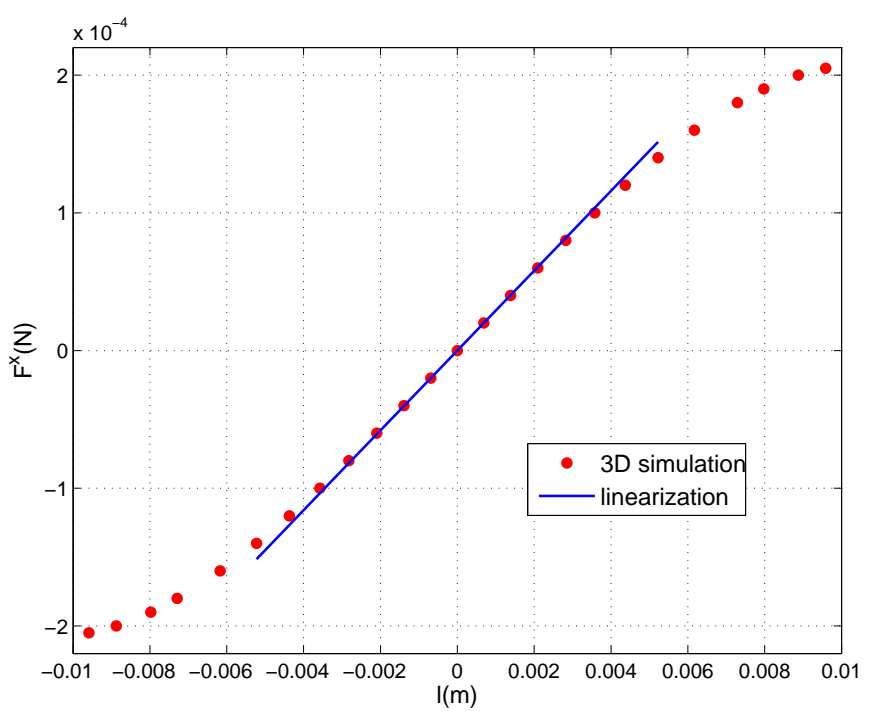

Figure 5: Simulated steady-state characteristic of the external force $F^{x}$ versus the displacement $x$ of the maglevtube.

expected for the force is $0.75 \mathrm{nN}$ with a CL4 head and $0.12 \mathrm{nN}$ with a CL2 head. In practice, such small values can not be reached because of the seismic mass sensitivity to seismic disturbances (subsonic air disturbances can be avoided by enclosing the sensor with a chamber). Stochastic low frequency seismic vibrations of magnets $M_{1}$ and $M_{1}^{\prime}$ generate unwanted magnetic return forces that are applied on magnets $M_{2}$. The result is a small stochastic oscillating behaviour for the maglevtube. All the forces induced by these environmental seismic and acoustic noises (see Fig. 1) along $\vec{x}$ axis are included in the force $F^{x}$ measured by the sensor. Thus care must be taken to reduce as much as possible this environmental noise. With a massive concrete ground to minimise seismic vibrations, a closed chamber and a passive anti-vibration table, the minimal standard deviation currently reached for the maglevtube displacement is around $30 \mathrm{~nm}$ (measured with a CL2 head).

\section{Sensor modelling and calibration}

\subsection{One DOF linear modelling of the maglevtube dynamic}

Let $G$ the centre of gravity of the maglevtube and $x$ its position in the frame $R_{0}$ attached to its base (cf. Fig. 3). The frame $R_{0}$ is supposed to be a Galilean reference frame, which implies that all ground vibrations are filtered by an antivibration table (no acceleration). This hypothesis will be later discussed in Section 6.1. If an external force $F^{x}$ is applied under the assumption done in Eq. (2) to the maglevtube tip, the dynamic of $G$ along $\vec{x}$ can be modelled by [12]:

$$
m \ddot{x}=F^{x}+F_{m a g}^{x}+F_{v i s c}^{x}
$$

in which $F_{v i s c}^{x}$ is the viscous friction force (mainly due to the air friction against the rear deflector) and $F_{m a g}^{x}$ is the 


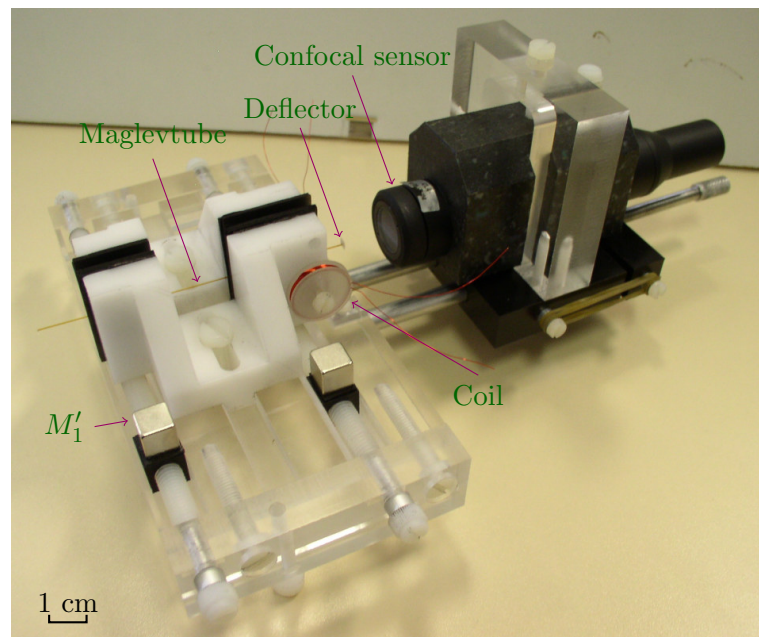

Figure 6: Force sensor prototype.

return magnetic force along $\vec{x}$ (magnetic spring). The diamagnetic force is completely negligible along $\vec{x}$. The force $F^{x}$ represents the sum of all others external forces applied on the tip along $\vec{x}$. It includes all the environmental disturbance forces along $\vec{x}$. Coordinate $x$ is set to zero when the maglevtube is in steady-state with $F^{x}$ set to zero. If the displacement of the tube along axis $\vec{x}$ remains in the linear domain given in Section 2.2 and if the speed is small, Eq. (4) becomes:

$$
m \ddot{x}=F^{x}-K_{m}^{x} x-K_{v}^{x} \dot{x}
$$

in which $K_{m}^{x}$ is the magnetic stiffness and $K_{v}^{x}$ the viscous damping coefficient. A possible state equation associated to Eq. (5) with $F^{x}(t)$ as input, $x(t)$ as output and $X(t)=$ $\left[\begin{array}{ll}x & \dot{x}\end{array}\right]^{\top}$ as state is:

$$
\begin{gathered}
\dot{X}(t)=A X(t)+B F^{x}(t) \\
x(t)=C X(t) \\
A=\left[\begin{array}{cc}
0 & 1 \\
-\frac{K_{m}^{x}}{m} & -\frac{K_{v}^{x}}{m}
\end{array}\right] \quad B=\left[\begin{array}{l}
0 \\
\frac{1}{m}
\end{array}\right] \quad C=\left[\begin{array}{ll}
1 & 0
\end{array}\right]
\end{gathered}
$$

\subsection{Calibration}

Calibration is usually a complex problem for micro and nanoforce sensors based on elastic microstructures because of the lack of standard forces at this scale. Stiffness absolute uncertainty is most of the time not specified and is still an open question on which are working international metrology laboratories [14]. Calibrating micro force sensors based on macroscopic seismic mass is easier and several dynamic calibration methods have been investigated. They are based on particular external force generation like impact force [15], step force [16] and oscillating force $[17,18]$. Because the maglevtube mass $m$ can be easily measured with a precision balance, a Zero Input Response (ZIR) is another possible way to identify the two

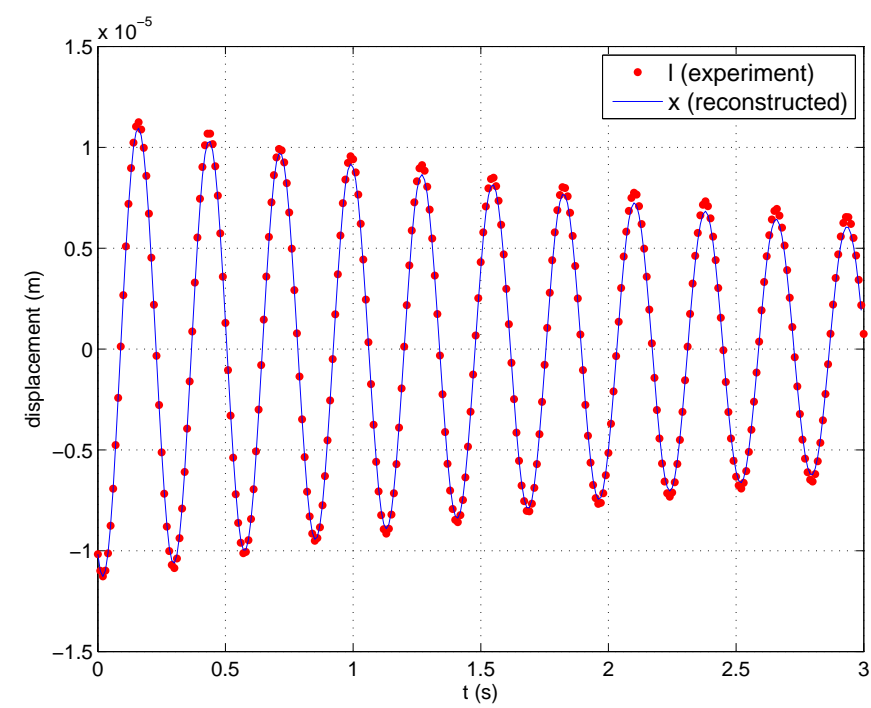

Figure 7: Measured and reconstructed zero input response (ZIR) of the maglevtube displacement.

others parameters $K_{m}^{x}$ and $K_{v}^{x}$ with an ARMA identification process. It requires an unknown excitation force $F^{x}$ with the following dynamic:

$$
\begin{array}{ll}
F^{x}(t) \neq 0 & t_{0} \leq t<t_{1} \quad \forall F^{x} \\
F^{x}(t)=0 & t \geq t_{1} .
\end{array}
$$

The output $x$ after $t_{1}$ is the ZIR of the maglevtube with unknown initial conditions (position and speed of the maglevtube at $\left.t_{1}\right)$. The temporal shape of $F^{x}(t)$ before $t_{1}$ necessary to excite the maglevtube does not matter (see Eq. (9a)) but must be equal to zero after $t_{1}$ (see Eq. (9b)). For the identification process, the behaviour of the maglevtube is taken into account only for time $t$ superior to $t_{1}$ and is therefore a ZIR. In the prototype, $F^{x}(t)$ is generated using two coils located near the rear diamagnetic plates (see Fig. 6). Nevertheless in practice, Eq. (9b) can not be completely verified because of the environmental disturbance forces that always acts on the maglevtube (these forces can be considered as a noise on the input. Its amplitude is between $\pm 1 \mathrm{nN}$ (see Fig. 22) and the dynamic it induces needs to be negligible. This condition is achieved with an excitation of the maglevtube enough high). The acquisition of $x$ starts at $t_{1}$ with a sampling time set to 0.01 seconds. Knowing the mass $m$, Fig. 7 shows the matching between both experimental ZIR and the continuous linear model after the parametric identification of $K_{m}^{x}$ and $K_{v}^{x}$ in Eq. (5) when $F^{x}$ is set to zero (done with Matlab ARMA identification). The frequency response of the identified linear model is shown in Fig. 8. Resonant frequency in displacement is around 3 Hertz. The low $-3 \mathrm{~dB}$ cutoff frequency (around $4 \mathrm{Hertz}$ ) will limit the resulting bandwidth during force measurements even if the force bandwidth can be reasonably extended to 20 Hertz because of the behaviour of the Kalman filter (see Section 5). 


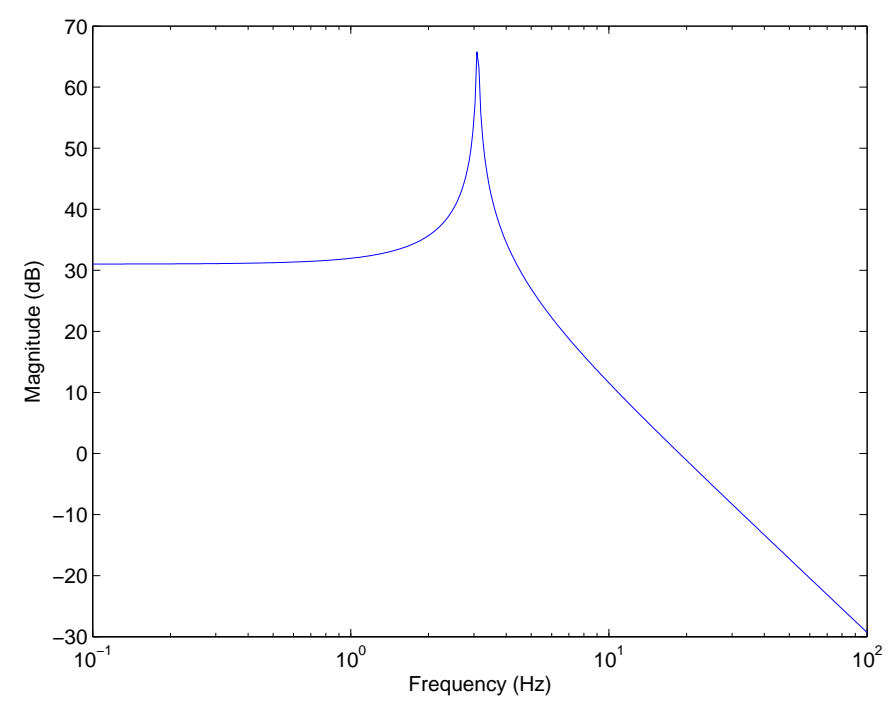

Figure 8: Simulated frequency response along $x$ of the identified linear model.

\section{Unknown input force estimation}

Despite the fact that the model-based deconvolution framework of a noisy output is an ill-posed problem with no exact solution, numerous approaches have been developed in the past such as for instance the Wiener deconvolution filter or deconvolution methods based on regularization [19]. In the specific context of micro or nanoforce measurement in microrobotics, the deconvolution problematic has been little addressed but some alternative approaches using unknown input observers have been recently published [20]. These approaches generally requires to set several parameters and the level of noise in the input reconstructed can not be adjusted. The method proposed here only requires one parameter in order to adjust an intuitive trade-off (like in regularization methods) between the wished resolution of the force estimation (this point sets the level of noise in the force estimation) and the response time of the estimation.

\subsection{A priory force modelling}

The noisy measurement $m_{k}^{x}$ of the maglevtube displacement along axis $x$ with the confocal chromatic sensor is done with a sampling period $T_{s}$ at each sampling time $t_{k}=k T_{s}$. The estimation of the unknown force with the set $\left\{m_{k}^{x}\right\}_{k \geq 0}$ is done at each sampling time $t_{k}$. It is based on an a priori discrete-time stochastic model for the force evolution that will be processed inside a recursive discrete Kalman filter. In this approach, the maglevtube model is deterministic. The stochastic part only concerns the modelling of the uncertainty associated to the unknown input force and the modelling of the noise generated by the confocal chromatic sensor.

The working out of the a priori uncertain model used to represent the force evolution is based on a stochastic
Wiener process:

$$
\dot{F}(t)=\omega(t)
$$

$F(t)$ in Eq. (10) is a model for the real force $F^{x}(t)$ and $\omega(t)$ is a zero-mean infinite-variance white Gaussian stochastic process representing the fact that the evolution of the force derivative is not known accurately. The autocorrelation function $\phi_{\omega, \omega}$ of this process is characterised by its power spectral density (PSD) $W_{\dot{F}}$ :

$$
\phi_{\omega, \omega}(\tau)=W_{\dot{F}} \delta(\tau) \quad \forall \tau \in \mathbb{R}
$$

The scalar term $W_{\dot{F}}$ is a parameter to set by the enduser that will influence in a given way the dynamic of the unknown force estimation (see Section 5). Because the process is white, all random variables $\omega(t)$ for any $t$ are independent and it is the same for the force increment between any time interval $\left|t_{i}-t_{j}\right|[21]$. Thus knowing the force increment between two instants gives absolutely no information on the increment between two other instants. As a consequence, this a priori model on the force evolution is very little informative.

To estimate the force with a state estimator it is necessary to merge the uncertain modelling of the input force with the deterministic model of the maglevtube in order to obtain the following extended state:

$$
X^{e}(t)=\left[\begin{array}{lll}
x & \dot{x} & F
\end{array}\right]^{\top}
$$

Its associated state-space model is obtained with Eqs. (10) and (6) in which the unknown input force $F^{x}(t)$ is replaced with the modelled random variable $F(t)$ :

$$
\begin{aligned}
\dot{X}^{e}(t) & =\mathcal{A} X^{e}(t)+\mathcal{M} \omega(t) \\
x(t) & =\mathcal{C} X^{e}(t)
\end{aligned}
$$

with

$$
\mathcal{A}=\left[\begin{array}{ccc}
A_{11} & A_{12} & B_{11} \\
A_{21} & A_{22} & B_{21} \\
0 & 0 & 0
\end{array}\right] \mathcal{M}=\left[\begin{array}{l}
0 \\
0 \\
1
\end{array}\right] \mathcal{C}=\left[\begin{array}{lll}
1 & 0 & 0
\end{array}\right]
$$

The state-space equation (13) is driven by the non-physical input $\omega(t)$ and thus by the parameter $W_{\dot{F}}$ to set (see Fig. 9).

To estimate with a discrete Kalman filter the extended state $X^{e}$ and thus the external force $F$ (third component of $\left.X^{e}\right)$ at each sampling time $t_{k}$, a discretization of Eqs. (13)(14) is necessary. The use of a zero-order hold $(\mathrm{ZOH})$ on $\omega(t)$ gives:

$$
\begin{aligned}
X_{k+1}^{e} & =\mathcal{F} X_{k}^{e}+\Omega_{k} \\
x_{k} & =\mathcal{C} X_{k}^{e}
\end{aligned}
$$

with:

$$
X_{k}^{e}=\left[\begin{array}{lll}
x_{k} & \dot{x}_{k} & F_{k}
\end{array}\right]^{\top} \quad \Omega_{k}=\left[\begin{array}{lll}
\omega_{k}^{x} & \omega_{k}^{\dot{x}} & \omega_{k}^{F}
\end{array}\right]^{\top}
$$



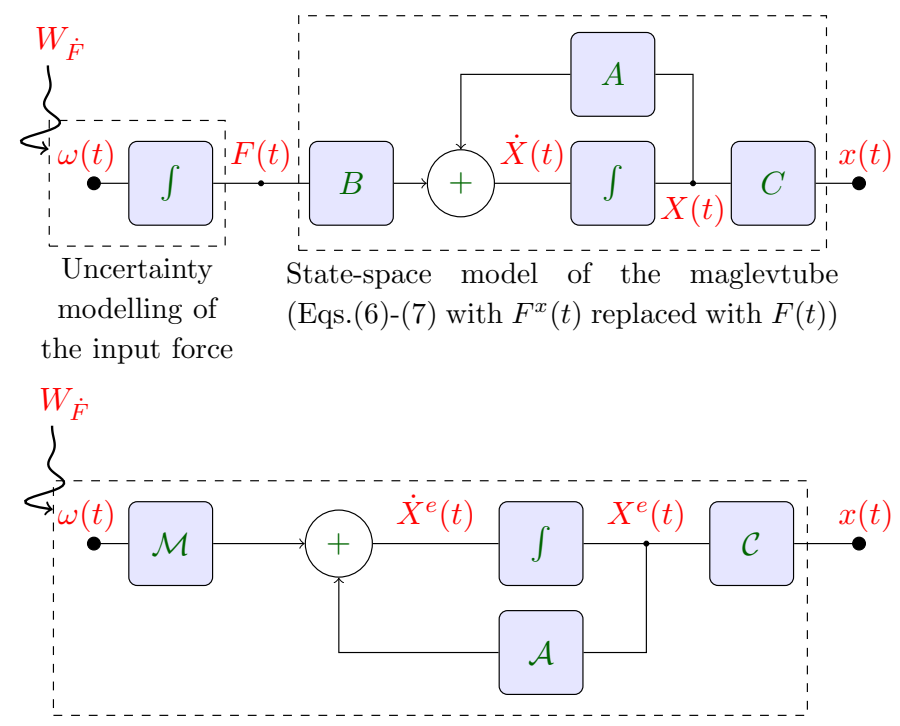

Associated extented state-space model (Eqs.(13)-(14))

Figure 9: Blocks representation of the uncertain modelling of the input force and the deterministic model of the maglevtube.

and

$$
\mathcal{F}=e^{\mathcal{A} T_{s}}=\left[\begin{array}{ccc}
\mathcal{F}_{11} & \mathcal{F}_{12} & \mathcal{F}_{13} \\
\mathcal{F}_{21} & \mathcal{F}_{22} & \mathcal{F}_{23} \\
0 & 0 & 1
\end{array}\right]
$$

The zero-mean white Gaussian process noise $\Omega_{k}$ characterises uncertainties on $x_{k}, \dot{x}_{k}$ and $F_{k}$ due to the stochastic force model used and to the discretization of the maglevtube dynamic. Its $3 \times 3$ covariance matrix $Q$ is:

$$
\begin{aligned}
Q=E\left[\Omega_{k} \Omega_{k}^{\top}\right] & =\int_{0}^{T_{s}} e^{\mathcal{A} t} \mathcal{M} W_{\dot{F}} \mathcal{M}^{\top} e^{\mathcal{A}^{\top} t} d t \\
& =W_{\dot{F}} \int_{0}^{T_{s}} e^{\mathcal{A} t} \mathcal{M} \mathcal{M}^{\top} e^{\mathcal{A}^{\top} t} d t \\
& =W_{\dot{F}} \eta\left(T_{s}\right)
\end{aligned}
$$

It appears with Eq. (22) that the matrix $Q$ is a tuning parameter derived from the scalar $W_{\dot{F}}$ and the sampling period $T_{s}$ that are both chosen by the end-user. Thus the matrix $Q$ is not associated to a physical process noise. This process noise $\Omega_{k}$ related to $X_{k}^{e}$ dynamic is only a mathematical consequence of the merging of the uncertain modelling of the input force with the deterministic model of the maglevtube followed by a discretization.

For a given sampling period $T_{s}$ in Eq. (22), $Q$ is proportional to $W_{\dot{F}}$ and it can be easily shown with Eq. (21) thanks to the specific shape of $e^{\mathcal{A} t}$ (see Eq. (19)) and $\mathcal{M}$ that the variance of $\omega_{k}^{F}$ (third component of $\Omega_{k}$ ) is equal to:

$$
\sigma^{2}\left(\omega_{k}^{F}\right)=Q_{33}=T_{s} W_{\dot{F}}
$$

The evolution of $F_{k}$ (third component of $X_{k}^{e}$ ) is obtained from Eqs. (16) and (18):

$$
F_{k+1}=F_{k}+\omega_{k}^{F} \quad k \geq 0
$$

Thus, if $W_{\dot{F}}$ is set to a given value, longer is the sampling period $T_{s}$ in Eq. (23) and bigger is the variance of $\omega_{k}^{F}$. That means in Eq. (24) that the uncertainty in the force evolution is larger. This is coherent with the fact that the longer sampling period will gives less information on the force evolution.

The statistic properties of the random process $\omega_{k}^{F}$ are:

$$
\begin{aligned}
& E\left[\omega_{k}^{F}\right]=\mu\left(\omega_{k}^{F}\right)=0 \quad \forall k \\
& E\left[\left(\omega_{k}^{F}-\mu\left(\omega_{k}^{F}\right)\right)^{2}\right]=\sigma^{2}\left(\omega_{k}^{F}\right)=T_{s} W_{\dot{F}} \\
& E\left[\omega_{i}^{F} \omega_{j}^{F}\right]=\sigma^{2}\left(\omega_{i}^{F}\right) \delta_{i j}=T_{s} W_{\dot{F}} \delta_{i j}
\end{aligned}
$$

Eqs. (24) to (27) fully characterise the a priori discretetime Gaussian stochastic model on the force evolution that will be used inside the Kalman filter. The uncertainty model represented by Eq. (24) corresponds to a discretetime Gaussian random walk that is usually written as follow (index shift on $\omega_{k}^{F}$ ):

$$
F_{k}=F_{k-1}+\omega_{k}^{F} \quad k \geq 1
$$

It comes from Eq. (28) that:

$$
F_{k}=\sum_{i=1}^{k} \omega_{i}^{F}+F_{0}
$$

Because successive random variables $\omega_{i}^{F}$ form an a priori discrete zero-mean white Gaussian process (the white property is induced by Eq. (27)), $F_{k}$ in Eq. (29) is Gaussian if the uncertainty on $F_{0}$ is assumed Gaussian or if $F_{0}$ is supposed equal to some fixed value. Its a priori variance at each step $k$ can be calculated thanks to Eq. (27):

$$
\begin{aligned}
\sigma^{2}\left(F_{k}\right) & =\sum_{i=1}^{k} \sigma^{2}\left(\omega_{i}^{F}\right)+\sigma^{2}\left(F_{0}\right) \\
& =k T_{s} W_{\dot{F}}+\sigma^{2}\left(F_{0}\right) \\
& =t_{k} W_{\dot{F}}+\sigma^{2}\left(F_{0}\right)
\end{aligned}
$$

Eq. (32) shows that bigger is the parameter $W_{\dot{F}}$ to set and bigger is the a priori uncertainty (variance) of the modelled unknown force $F_{k}$ at time $t_{k}$. The uncertainty growth in time is also linear with $t_{k}$ (see Fig. 10). Let us now assuming that the a priori uncertainty in the future on the value of a rapidly varying unknown force is necessarily higher than for a slowly varying unknown force. In this case, the model given by Eq. (32) involves that the parameter $W_{\dot{F}}$ should be set higher for a rapidly varying force than for a slowly varying force. This primary qualitative analysis on the choice of $W_{\dot{F}}$ will be completed in Section 5 .

Finally, it is necessary to take into account the fact that the measurement $m_{k}^{x}$ of $x_{k}$ is obtained with a confo- 


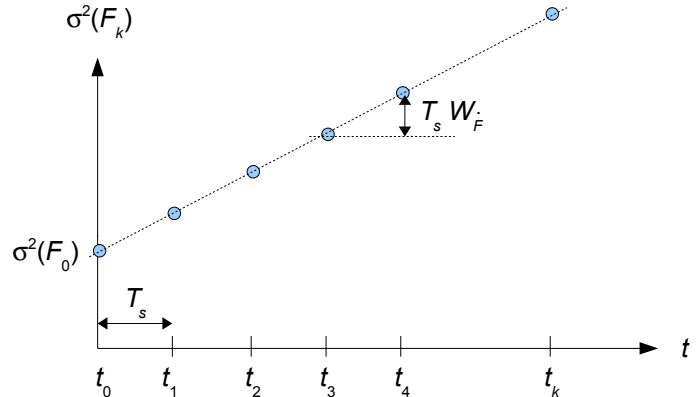

Figure 10: $W_{\dot{F}}$ effect on the a priori variance $\sigma^{2}\left(F_{k}\right)$.

cal chromatic sensor that adds to $x_{k}$ a discrete-time bandlimited white Gaussian noise $v_{k}$ with zero-mean and variance $R$ :

$$
m_{k}^{x}=x_{k}+v_{k}
$$

Eq. (33) combined with Eqs. (16)-(17) gives the state-space model with output $m_{k}^{x}$ that will be used by the discrete Kalman filter:

$$
\begin{aligned}
X_{k+1}^{e} & =\mathcal{F} X_{k}^{e}+\Omega_{k} \\
m_{k}^{x} & =\mathcal{C} X_{k}^{e}+v_{k}
\end{aligned}
$$

with

$$
\begin{array}{ll}
E\left[\Omega_{k}\right]=0_{3 \times 1} & E\left[\Omega_{k} \Omega_{k}^{\top}\right]=Q=W_{\dot{F}} \eta\left(T_{s}\right) \\
E\left[v_{k}\right]=0 & E\left[v_{k}^{2}\right]=R
\end{array}
$$

\subsection{Force estimation using a time-varying Kalman filter}

Fig. 11 shows the force estimation using a Kalman filter. The input of the Kalman filter is the noisy measurement $m_{k}^{x}$ of the maglevtube displacement. The output is $\hat{F}_{k}$. If the parameter $W_{\dot{F}}$ is changed by the end-user during the force estimation process, a time-varying Kalman filter must be used and a numerical computation of $Q$ must be done each time $W_{\dot{F}}$ is changed (nevertheless, the term $\eta\left(T_{s}\right)$ in Eq. (22) can be pre-calculated for a given sampling period $\left.T_{s}[22]\right)$. The prediction-estimation stages of the Kalman filter are derived from Eqs. (16) and (35). The prediction stage is:

$$
\begin{aligned}
& \hat{X}_{k \mid k-1}^{e}=\mathcal{F} \hat{X}_{k-1}^{e} \\
& P_{k \mid k-1}=\mathcal{F} P_{k-1} \mathcal{F}^{\top}+Q
\end{aligned}
$$

with $P_{k \mid k-1}$ the covariance of the prediction error conditioned by all previous measurements done before time $t_{k}$ :

$$
\begin{aligned}
P_{k \mid k-1} & =E\left[\left(X_{k}^{e}-\hat{X}_{k \mid k-1}^{e}\right)\left(X_{k}^{e}-\hat{X}_{k \mid k-1}^{e}\right)^{\top} \mid M_{k-1}\right] \\
M_{k-1} & =\left\{m_{1}^{x}, \ldots, m_{k-1}^{x}\right\}
\end{aligned}
$$

In Eq. (38), the third component of $\hat{X}_{k \mid k-1}^{e}$ is the force prediction $\hat{F}_{k \mid k-1}$ that remains equal to the last estimation:

$$
\hat{F}_{k \mid k-1}=\hat{F}_{k-1}
$$

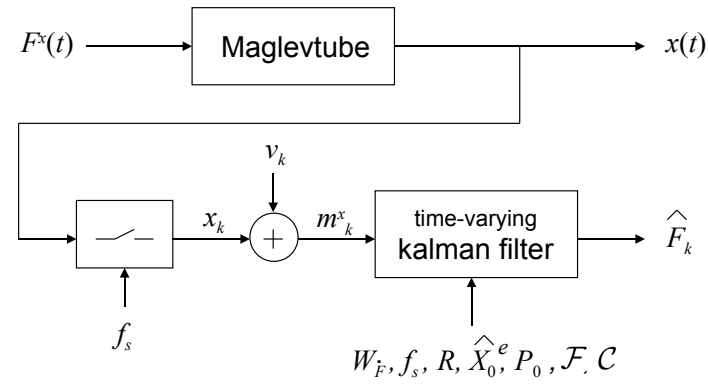

Figure 11: Force estimation with a time-varying Kalman filter

The estimation stage that gives $\hat{F}_{k}$ is:

$$
\begin{aligned}
K_{k} & =P_{k \mid k-1} \mathcal{C}^{\top}\left(\mathcal{C} P_{k \mid k-1} \mathcal{C}^{\top}+R\right)^{-1} \\
\hat{X}_{k}^{e} & =\hat{X}_{k \mid k-1}^{e}+K_{k}\left(m_{k}^{x}-\mathcal{C} \hat{X}_{k \mid k-1}^{e}\right) \\
\hat{F}_{k} & =\mathcal{C}_{F} \hat{X}_{k}^{e} \\
P_{k} & =\left(I_{3 \times 3}-K_{k} \mathcal{C}\right) P_{k \mid k-1}
\end{aligned}
$$

with $P_{k}$ the conditional covariance of the estimation error defined by:

$$
\begin{aligned}
P_{k} & =E\left[\left(X_{k}^{e}-\hat{X}_{k}^{e}\right)\left(X_{k}^{e}-\hat{X}_{k}^{e}\right)^{\top} \mid M_{k}\right] \\
M_{k} & =\left\{m_{1}^{x}, \ldots, m_{k-1}^{x}, m_{k}^{x}\right\}
\end{aligned}
$$

and $\mathcal{C}_{F}$ the output matrix giving the estimated force:

$$
\mathcal{C}_{F}=\left[\begin{array}{lll}
0 & 0 & 1
\end{array}\right]
$$

The Kalman filter is a priori initialised for instance with the maglevtube in its equilibrium state when no force is applied to it:

$$
\hat{X}_{0}^{e}=E\left[X_{0}^{e}\right]=\left[\begin{array}{lll}
0 & 0 & 0
\end{array}\right]^{\top}
$$

The covariance matrix $P_{0}$ of the initial estimation error is taken equal to:

$$
\begin{aligned}
P_{0} & =E\left[\left(X_{0}^{e}-\hat{X}_{0}^{e}\right)\left(X_{0}^{e}-\hat{X}_{0}^{e}\right)^{\top}\right] \\
& =\left[\begin{array}{ccc}
\sigma^{2}\left(x_{0}\right) & 0 & 0 \\
0 & \sigma^{2}\left(\dot{x}_{0}\right) & 0 \\
0 & 0 & \sigma^{2}\left(F_{0}\right)
\end{array}\right]
\end{aligned}
$$

In Eq. (51), each variance on the main diagonal represents the a priori uncertainty on $x_{0}, \dot{x}_{0}$ and $F_{0}$. The term $\sigma^{2}\left(F_{0}\right)$ is shown in Fig. 10. These values are chosen to be coherent with the initial conditions associated to the experiment made. In practice, they have little importance if the user starts the Kalman filter with no force applied on the maglevtube and waits a few seconds such that the Kalman gain $K_{k}$ converges to its steady-state $K_{\infty}$ (solution to the discrete Riccati equation that depends on $W_{\dot{F}}$, $f_{s}$ and $R$ ) before applying an unknown varying external force. Each time $W_{\dot{F}}$ is changed, $K_{\infty}\left(W_{\dot{F}}, f_{s}, R\right)$ evolves to a new value. 


\section{Analysis of simulated input forces}

\subsection{Estimation of a force generated by a Wiener Process}

The study in simulation of the estimation behaviour is first conducted with an external force $F^{x}(t)$ generated by a Wiener process (like Eq. (10)) considered as a shaping filter instead of an uncertainty model:

$$
\dot{F}^{x}(t)=\omega_{r}(t)
$$

This specific case corresponds to a brownian evolution of the input force $F^{x}(t)$. Its interest is to observe qualitatively what happens if the parameter $W_{\dot{F}}$ is not equal to the PSD $W$ of the zero-mean white Gaussian process $\omega_{r}(t)$ :

$$
\phi_{\omega_{r}, \omega_{r}}(\tau)=W \delta(\tau) \quad \forall \tau \in \mathbb{R}
$$

The PSD $W_{\dot{F}}$ can be set equal, inferior or superior to $W$ by the end-user who does not know the real value of $W$. Sampling frequency $f_{s}$ is 100 Hertz. The variance $R$ of the measurement noise is $1.44 \times 10^{-16} \mathrm{~m}^{2}$ (typical value for a CL2 confocal head provided by STIL SA).

Fig. 12 shows $F^{x}(t)$ and $\hat{F}_{k}$ when $W_{\dot{F}}$ is chosen equal to $W$ and both set to $10^{-14} \mathrm{~N}^{2} / \mathrm{Hz}$. The maglevtube displacement that remains in the linear domain is also plotted for reference. In this case, the error estimation between the sampled input force and $\hat{F}_{k}$ has a minimal variance conditionally to the measurements done (classical framework of Kalman filtering). In Fig. 12(a), a slight smoothing induced by the sampling period $T_{s}$ can be seen between $\hat{F}_{k}$ and the non-sampled force $F(t)$.

Fig. 13(a) shows $F^{x}(t)$ and $\hat{F}_{k}$ if $W_{\dot{F}}$ is inferior to $W$ (the last one is still equal to $10^{-14} \mathrm{~N}^{2} / \mathrm{Hz}$ ). This choice of $W_{\dot{F}}$ leads to a covariance matrix $Q$ that does not reflect the real covariance matrix associated to the sampled force $F^{x}(t)$. In this case, $Q$ can be seen as a parameter that influence the estimation dynamic in some ways that will be studied in the next Sections. The estimation is then much more smoothed and delayed (and it is not optimal in the sense of minimal variance). In Fig. 13(b) $W_{\dot{F}}$ is chosen superior to $W$ and the estimation is more noisy. Thus a primary observation is that $W_{\dot{F}}$ has an effect on the resulting force bandwidth (smoothing), on the delay and also on the noise present in the estimation.

\subsection{Estimation of a step input force}

Because the real force $F^{x}(t)$ does not follow generally the uncertain model given by Eq. (10), the behaviour of the deconvolution block must be characterised with criterions well adapted to SISO transfer functions. This characterisation will start by a temporal analysis of the effect of $W_{\dot{F}}$ on the estimation of a canonical step input force $F^{x}(t)$. This analysis will result in the study of a tradeoff between the resolution and the bandwidth of the force sensor.

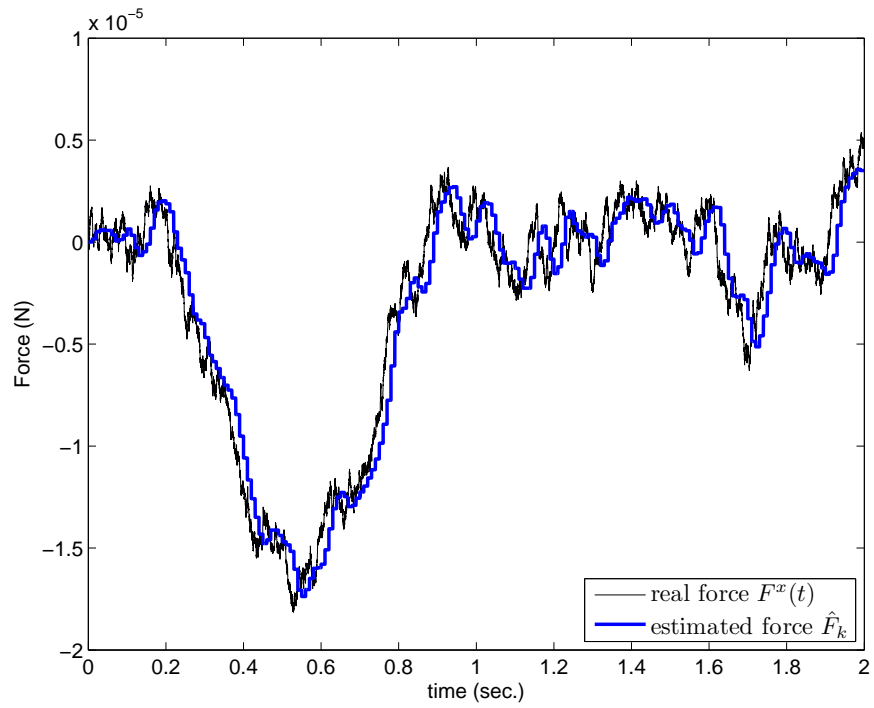

(a) Real and estimated force.

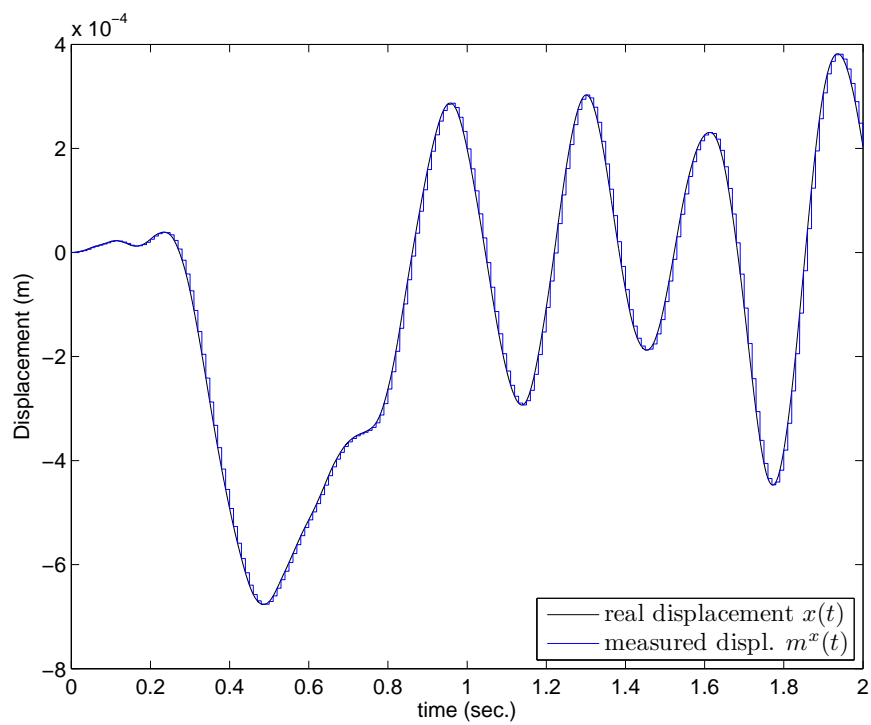

(b) Associated measured displacement.

Figure 12: Force estimation if $W=W_{\dot{F}}=10^{-14} \mathrm{~N}^{2} / \mathrm{Hz}$. 


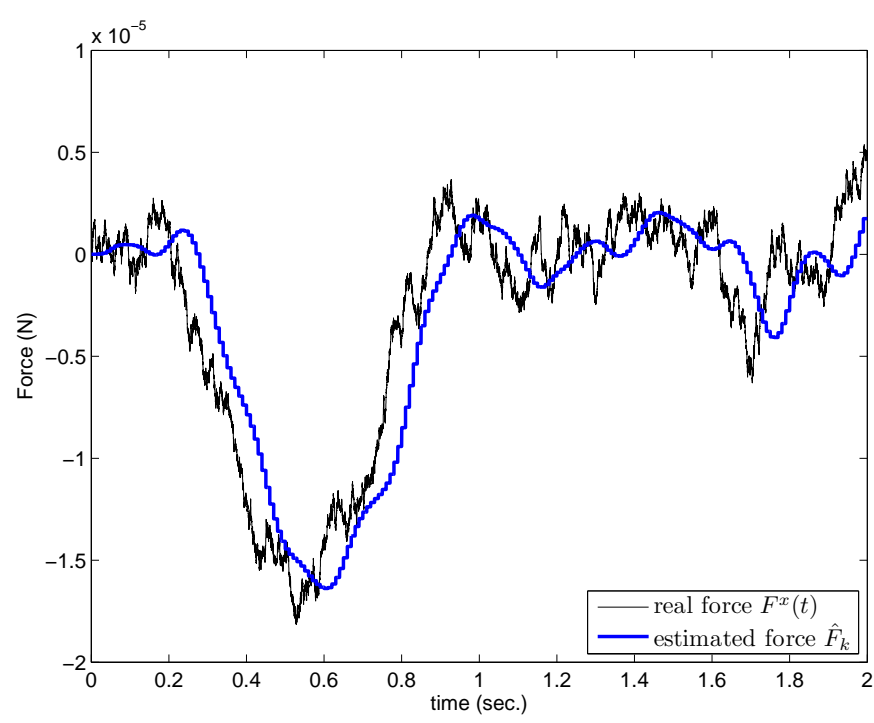

(a) Case $W_{\dot{F}}<W\left(W_{\dot{F}}=W / 1000\right)$.

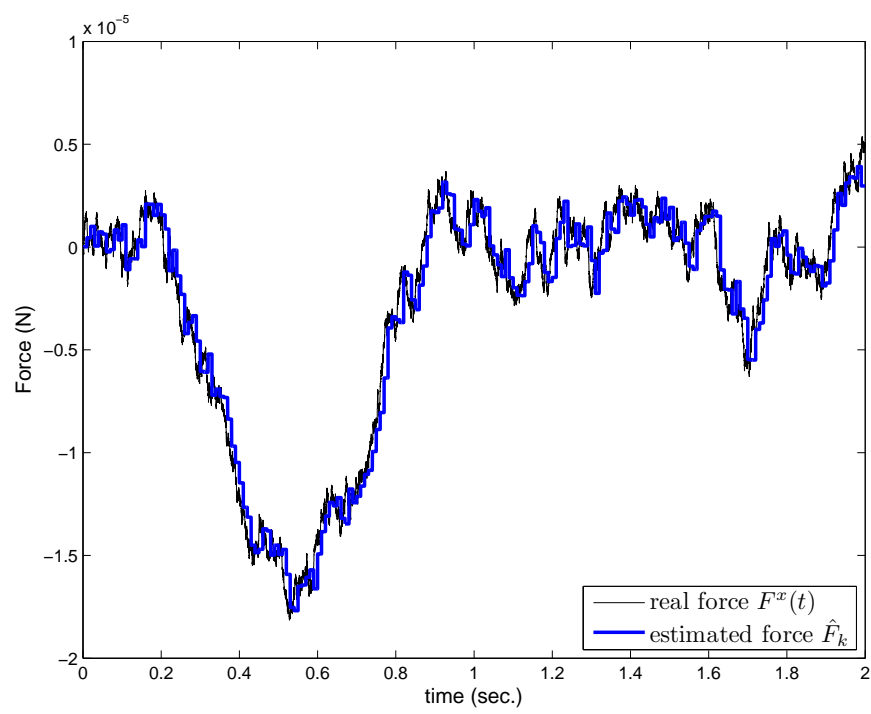

(b) Case $W_{\dot{F}}>W\left(W_{\dot{F}}=W \times 1000\right)$.

Figure 13: Real and estimated force if $W_{\dot{F}}$ is different from $W$ equal to $10^{-14} \mathrm{~N}^{2} / \mathrm{Hz}$.
The step amplitude is set to $100 \mathrm{nN}$ in all simulations. To be independent of $P_{0}$ specified in Eq. (51), a steadystate Kalman filter is used substituting $K_{\infty}\left(W_{\dot{F}}, f_{s}, R\right)$ to $K_{k}$ and using only Eqs. (38) (44) (45). The associated third-order state-space model (with measurement $m_{k}^{x}$ as input and estimation $\hat{F}_{k}$ as output) is obtained with Eq. (44) reported in Eq. (38) and using Eq. (45):

$$
\begin{aligned}
\hat{X}_{k+1 \mid k}^{e} & =A^{K} \hat{X}_{k \mid k-1}^{e}+B^{K} m_{k}^{x} \\
\hat{F}_{k} & =C^{K} \hat{X}_{k \mid k-1}^{e}+D^{K} m_{k}^{x}
\end{aligned}
$$

with

$$
\begin{aligned}
A^{K} & =\mathcal{F}\left(I_{3 \times 3}-K_{\infty} \mathcal{C}\right) & B^{K} & =\mathcal{F} K_{\infty} \\
C^{K} & =\mathcal{C}_{F}\left(I_{3 \times 3}-K_{\infty} \mathcal{C}\right) & D^{K} & =\mathcal{C}_{F} K_{\infty}
\end{aligned}
$$

The variance $R$ of the measurement noise remains equal to $1.44 \times 10^{-16} \mathrm{~m}^{2}$. The maglevtube parameters are $m=$ $74 \times 10^{-6} \mathrm{~kg}, K_{m}^{x}=0.02818 \mathrm{~N} / \mathrm{m}$ (in linear domain), $K_{v}^{x}=1.8 \times 10^{-5} \mathrm{~N} . \mathrm{s} / \mathrm{m}$ (the damping coefficient $\zeta$ is $\left.6.23 \times 10^{-3}\right)$. Identified values used in the Kalman filter are $K_{m}^{x}=0.02812 \mathrm{~N} / \mathrm{m}, K_{v}^{x}=1.772 \times 10^{-5} \mathrm{~N} . \mathrm{s} / \mathrm{m}(\zeta$ is $\left.6.14 \times 10^{-3}\right)$. The sampling frequency $f_{s}=1 / T_{s}$ is deliberately set to a high value $\left(f_{s}=1000\right.$ Hertz $)$ compared to the $-3 \mathrm{~dB}$ cutoff frequency of the maglevtube (around 4 Hertz) in order to see the effects of this choice.

Fig. 14 shows the force $\hat{F}_{k}$ estimated for $W_{\dot{F}}$ set to $10^{-18} \mathrm{~N}^{2} / \mathrm{Hz}$ and $10^{-15} \mathrm{~N}^{2} / \mathrm{Hz}$. In the last case, the response time is around 0.1 second and thus much shorter than the Maglevtube settling time at 5\% (20 seconds). Smaller is $W_{\dot{F}}$ and smaller is the noise on $\hat{F}_{k}$ but longer is the estimation response time. As a consequence, smaller is the amplitude to estimate and smaller must be $W_{\dot{F}}$ to have a good signal to noise ratio in $\hat{F}_{k}$. But in this case, the force bandwidth of the sensor is also reduced.

\subsection{Harmonic frequency response of the Kalman filter}

The previous behaviour can be explained with the frequency response of the deconvolution block which is the steady-state Kalman filter:

$$
\begin{aligned}
H^{K}(\omega) & =\frac{\hat{F}\left(e^{j \omega}\right)}{m^{x}\left(e^{j \omega}\right)} \\
& =C^{K}\left(e^{j \omega} I_{3 \times 3}-A^{K}\right)^{-1} B^{K}+D^{K}
\end{aligned}
$$

As it is shown in Fig. 15, this frequency response "inverts" the frequency response of the maglevtube with its resonance peak (see Fig. 8). Fig. 15 shows that bigger is $W_{\dot{F}}$ and bigger is the gain in the high frequencies just after the antiresonance. Thus bigger is the amplification of the high frequency components in the measurement noise $v_{k}$ included in the input $m_{k}^{x}$. Nevertheless, whatever is $W_{\dot{F}}$ the gain in the highest frequencies below the Nyquist frequency $f_{s} / 2$ is always decreasing to limit the amplification of the highest frequencies in the input noise. To reduce the 


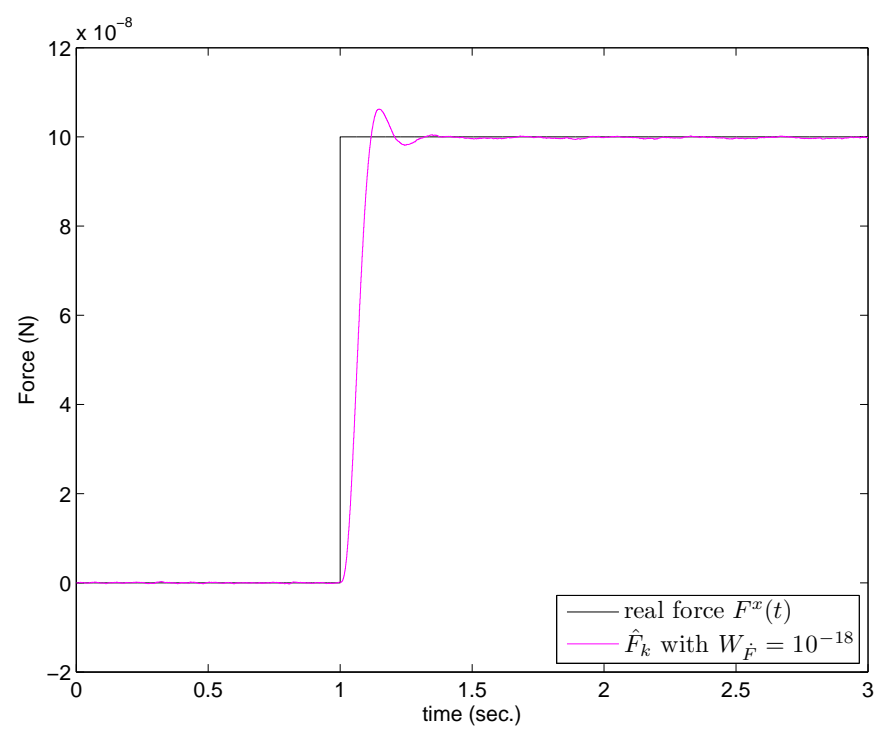

(a) Real and estimated force with $W_{\dot{F}}=10^{-18} \mathrm{~N}^{2} / \mathrm{Hz}$.

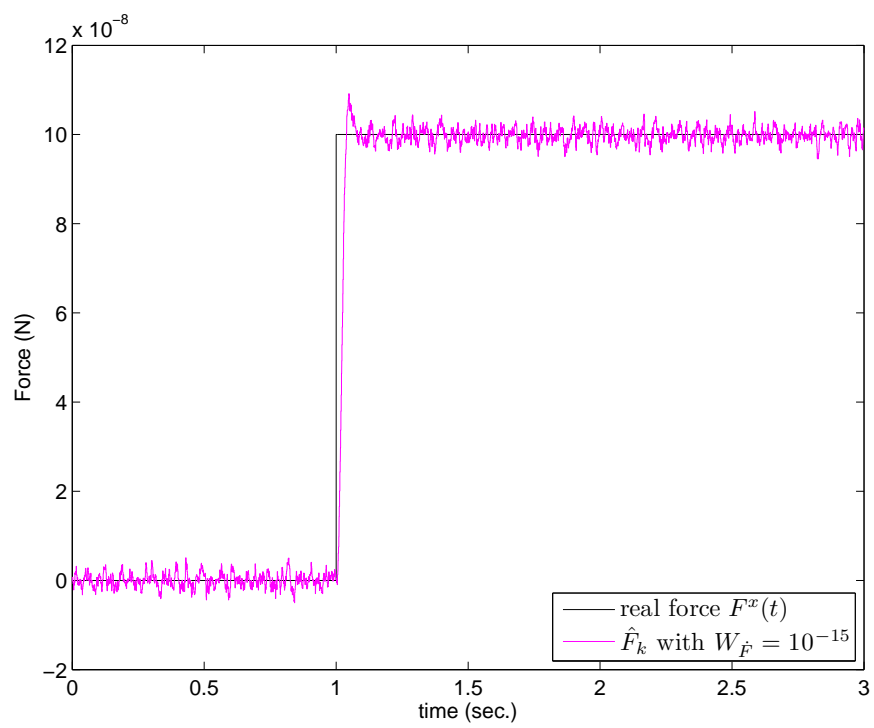

(b) Real and estimated force with $W_{\dot{F}}=10^{-15} \mathrm{~N}^{2} / \mathrm{Hz}$.

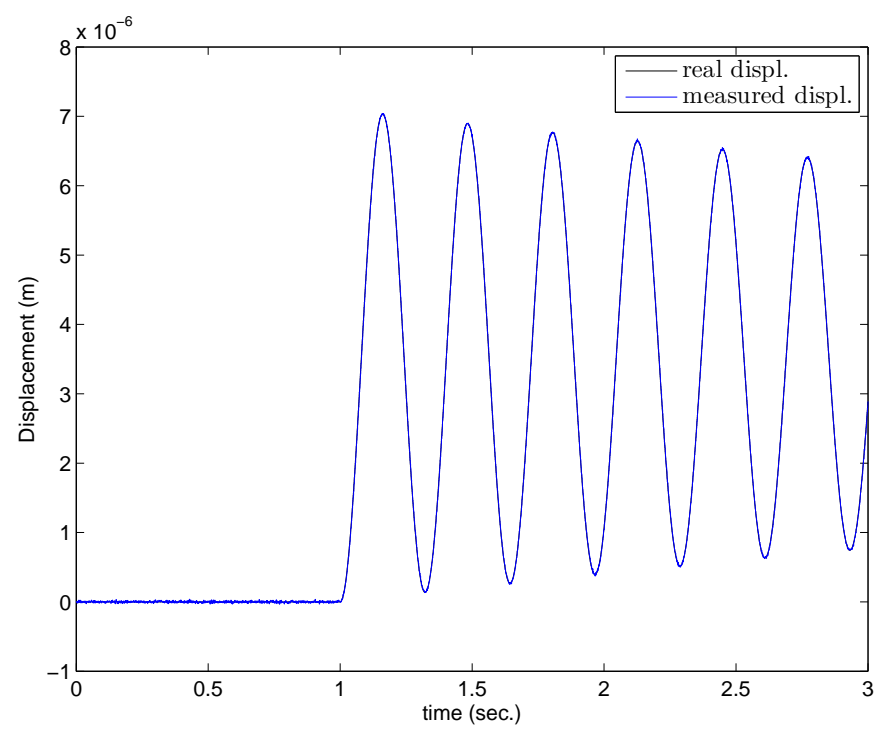

(c) Real and Measured displacement with $f_{s}=1000 \mathrm{~Hz}$.

Figure 14: Step force estimation with $F^{x}$ set to $100 \mathrm{nN}$. averall noise level, it is necessary to reduce $W_{\dot{F}}$. But in this case, the high frequency components of the displacement $m_{k}^{x}$ have a very small amplitude because the maglevtube acts as a low pass filter (see Fig. 8). These high frequencies are then insufficiently amplified by the Kalman filter (see Fig. 15(a) with $W_{\dot{F}}$ set to $10^{-18} \mathrm{~N}^{2} / \mathrm{Hz}$ ) to correctly reconstruct the high frequency components of the input $F(t)$ at the step time (time $t=1 \mathrm{sec}$ in Figs. 14(a) and 14(b)). As a consequence, the response time increases (and the bandwidth decreases). This behaviour is interesting because the maglevtube displacement becomes very small for high frequencies, especially if $f_{s}$ is set to a high value as it is deliberately the case here. As a consequence, the signal to noise ratio of the maglevtube displacement measurement is also bad in the high frequencies but the Kalman filter takes care of this information worsening by adjusting a trade-off (driven by $W_{\dot{F}}$ ) between the resolution of the estimated force ( $i e$ the noise level in $\hat{F}_{k}$ ) and the response time of the estimation.

\subsection{Force sensor resolution}

The additive coloured Gaussian noise $n_{k}$ presents in the estimation $\hat{F}_{k}$ (see Fig. 14(b) for instance) is the consequence of the measurement noise $v_{k}$ (present in the input $\left.m_{k}^{x}\right)$ going through the transfer function $H^{K}(z)$. Statistical properties of $n_{k}$ can be determined using the Kalman filter state-space model given by Eqs. (54)-(55). As it is shown in Fig. 16, thanks to additivity of linear systems, only input $v_{k}$ is considered instead of the sum $m_{k}^{x}=$ $x_{k}+v_{k}$, which gives the following stochastic state-space model:

$$
\begin{aligned}
\hat{X}_{k+1 \mid k}^{e} & =A^{K} \hat{X}_{k \mid k-1}^{e}+B^{K} v_{k} \\
n_{k} & =C^{K} \hat{X}_{k \mid k-1}^{e}+D^{K} v_{k}
\end{aligned}
$$

The noise $v_{k}$ is the input and the noise $n_{k}$ is the output. $X_{k \mid k-1}^{e}$ is a random state with mean $m_{k}$ and covariance matrix $S_{k}$ given by:

$$
\begin{aligned}
m_{k} & =E\left[\hat{X}_{k \mid k-1}^{e}\right]=\left(A^{K}\right)^{k} m_{0} \\
S_{k+1} & =E\left[\left(\hat{X}_{k+1 \mid k}^{e}-m_{k+1}\right)^{2}\right] \\
& =A^{K} S_{k} A^{K^{\top}}+B^{K} R B^{K^{\top}}
\end{aligned}
$$

with $R$ defined in Eq. (37). Taking into account the fact that $v_{k}$ is a zero-mean Gaussian noise and that $\hat{X}_{k \mid k-1}^{e}$ and $v_{k}$ are independent at time $t_{k}$, the mean $\mu_{k}$ and variance $\Sigma_{k}$ of the Gaussian noise $n_{k}$ in Eq. (61) are given by:

$$
\begin{aligned}
& \mu_{k}=E\left[n_{k}\right]=C^{K} m_{k}+D^{K} E\left[v_{k}\right]=C^{K} m_{k} \\
& \Sigma_{k}=E\left[\left(n_{k}-\mu_{k}\right)^{2}\right]=C^{K} S_{k} C^{K^{\top}}+D^{K} R D^{K^{\top}}
\end{aligned}
$$

Because of the sum $m_{k}^{x}=x_{k}+v_{k}$ on the input, any initial condition different from zero for the (deterministic) state $X_{k \mid k-1}^{e}$ will be associated to the input $x_{k}$. Thus, by 


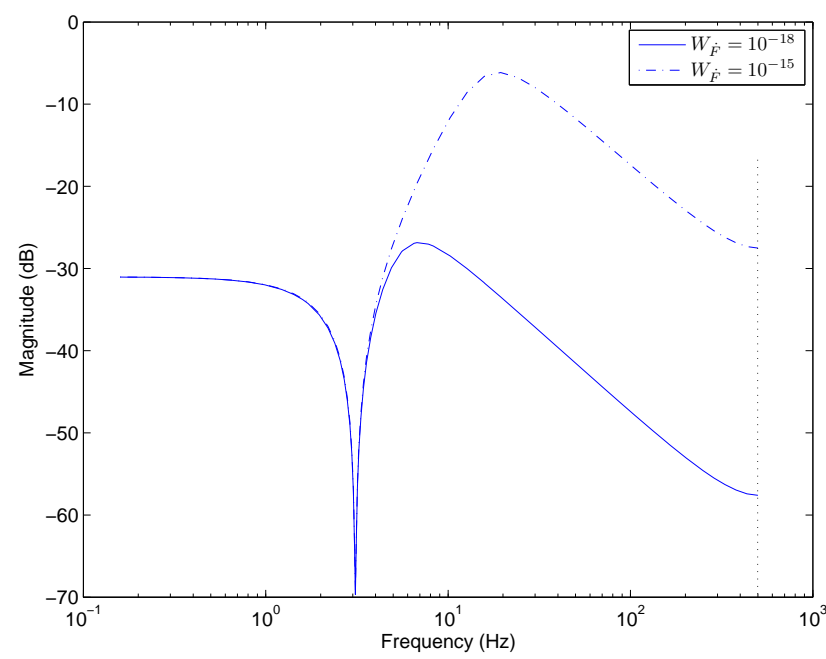

(a) Amplitude of $H^{K}(\omega)\left(f_{s}=1000 \mathrm{~Hz}\right)$.

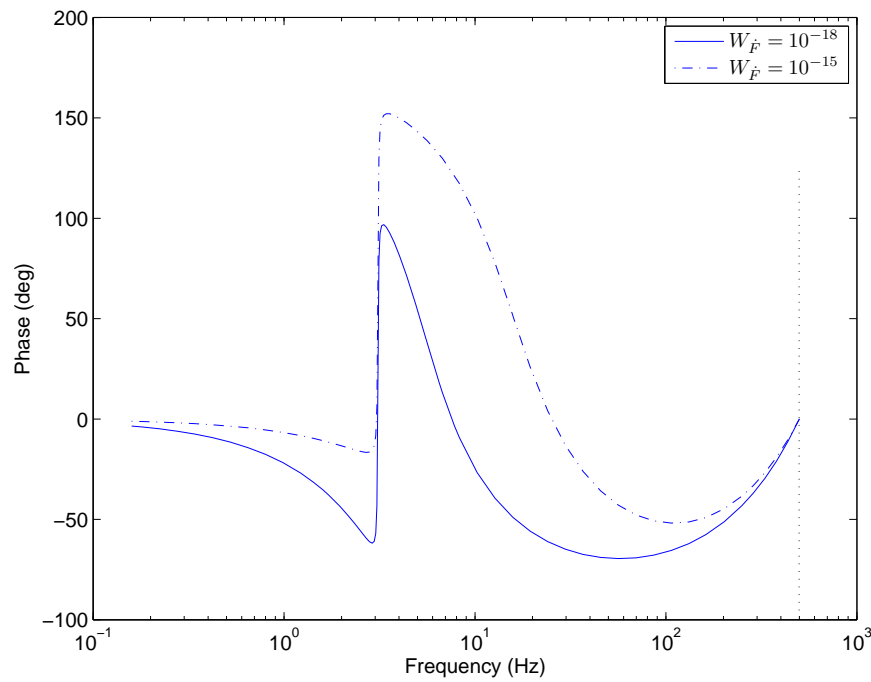

(b) Phase of $H^{K}(\omega)\left(f_{s}=1000 \mathrm{~Hz}\right)$.

Figure 15: Impact of $W_{\dot{F}}$ on the transfer function $\hat{F}\left(e^{j \omega}\right) / m^{x}\left(e^{j \omega}\right)$.

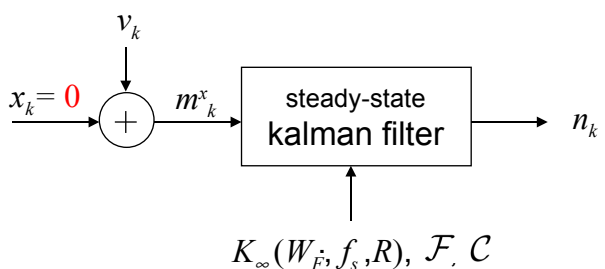

Figure 16: Output noise $n_{k}$ in estimation $\hat{F}_{k}$ due to the measurement noise $v_{k}$. additivity, it remains only a null initial condition for the (stochastic) state $X_{k \mid k-1}^{e}$ associated to the input $v_{k}$ :

$$
X_{0 \mid-1}^{e}=\left[\begin{array}{lll}
0 & 0 & 0
\end{array}\right]^{\top}
$$

Because the initial condition for this stochastic state $X_{k \mid k-1}^{e}$ is always the previous one, it comes:

$$
m_{0}=0_{3 \times 1} \quad S_{0}=0_{3 \times 3}
$$

Notice that $m_{0}$ and $S_{0}$ only influence the transient evolution of $n_{k}$ at the beginning of the measurement. With Eq. (62) the mean $m_{k}$ is always null and with Eq. (64) the statistical properties of $n_{k}$ are given by:

$$
\begin{aligned}
& \mu_{k}=0 \quad \forall k \\
& \Sigma_{k}=C^{K} S_{k} C^{K^{\top}}+D^{K} R D^{K^{\top}}
\end{aligned}
$$

The $99 \%$ confidence interval associated to Eq. (69) is plotted with red dotted lines in Fig. 17. The sampling frequency $f_{s}$ is set to 100 Hertz in this simulation. The mean in this Fig. corresponds to the value of $\hat{F}_{k}$ when there is no measurement noise $v_{k}$. This unknown value belongs to the confidence interval with a 0.99 probability. To avoid any misinterpretation, it does not mean that the real force $F^{x}(t)$ belongs to this interval, but only that $\hat{F}_{k}$ should belong to it if they were no measurement noise. The associated standard deviation $\sqrt{\Sigma_{k}}$ is an image of the resolution (or the SNR ratio) of the sensor. It tends to $\sqrt{\Sigma_{\infty}}$ in a few sampling times $t_{k}$ (see the first $0.05 \mathrm{sec}$ in Fig. 17). This value $\sqrt{\Sigma_{\infty}}$ can be given to the end-user to adjust $W_{\dot{F}}$. Bigger is $W_{\dot{F}}$ and bigger is $\sqrt{\Sigma_{\infty}}$ (see Fig. 18), thus lower is the resolution of the sensor. Variance $\Sigma_{k}$ has no connection to the force sensor accuracy. This last one only depends on the estimation of $m, K_{m}^{x}$ and $K_{v}^{x}$ during the calibration stage. In steady-state, the accuracy only depends on the calibration of $K_{m}^{x}$.

\subsection{Force sensor bandwidth}

The bandwidth of the force estimation depends on the harmonic transfer between the input force $F^{x}(t)$ and output estimation $\hat{F}_{k}$. Because time description is continuous for the input and discrete for the output, this transfer is difficult to model. Thus, it will be determined with only discrete time description. Because of this, the first transfer from $F^{x}(t)$ to $x(t)$ that represents the dynamic of the maglevtube is discretized with a zero-order hold with sampling period $T_{s}$ added on the input $F^{x}(t)$. This will leads to a slightly erroneous result when a pure sinusoidal input $F^{x}(t)$ is applied on the maglevtube.

The discretized linear state-space representation of the maglevtube is obtained from Eqs. (6) and (7):

$$
\begin{aligned}
X_{k+1} & =A_{d} X_{k}+B_{d} F_{k}^{x} \\
x_{k} & =C_{d} X_{k}
\end{aligned}
$$




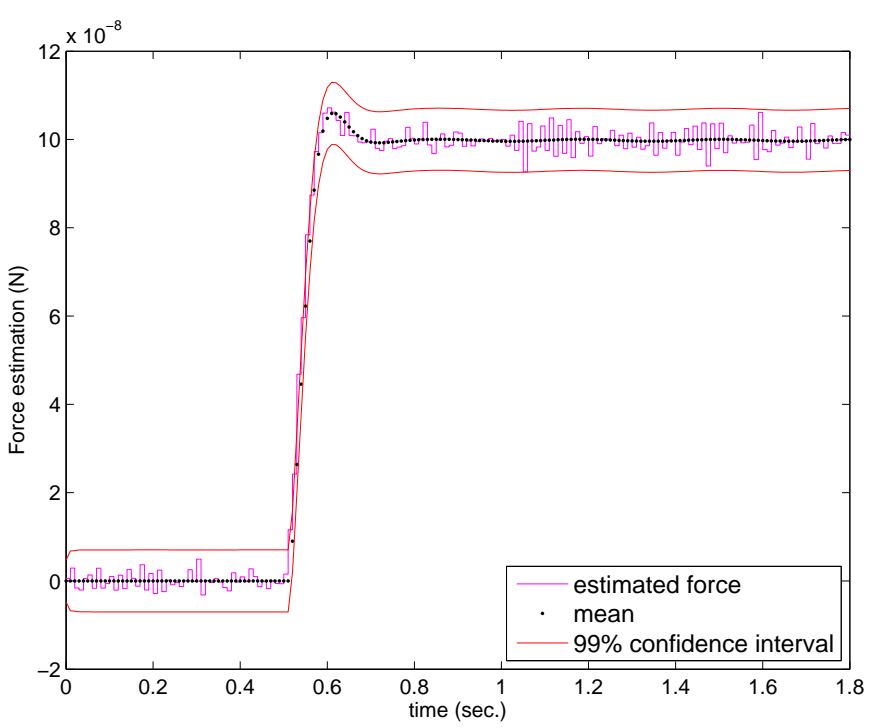

Figure 17: $99 \%$ confidence interval $\left( \pm 3 \sqrt{\Sigma_{k}}\right)$ on the force estimation with $W_{\dot{F}}=10^{-15} \mathrm{~N}^{2} / \mathrm{Hz}$ and $f_{s}=100 \mathrm{~Hz}$.

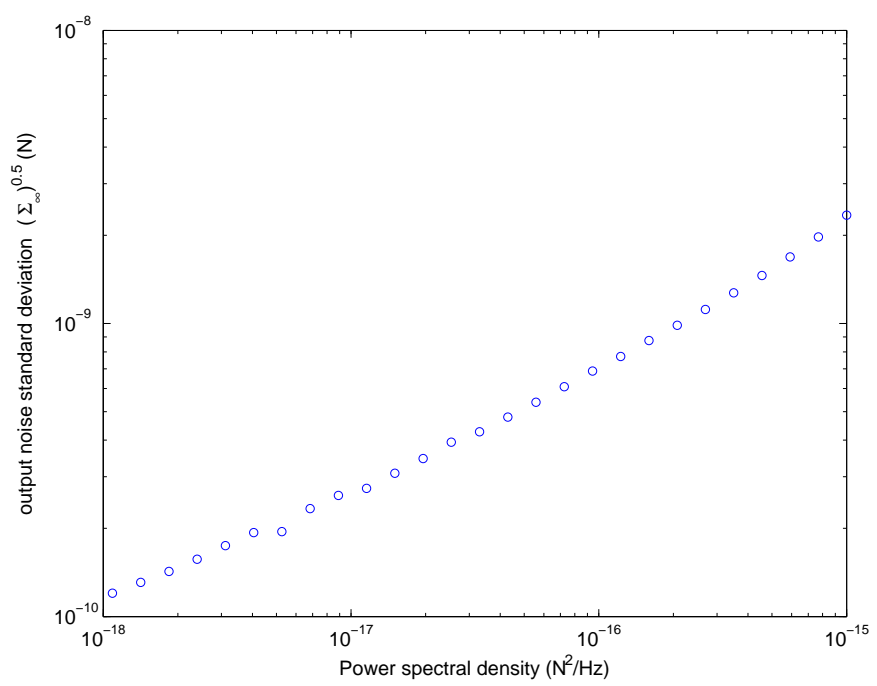

Figure 18: Standard deviation $\sqrt{\Sigma_{\infty}}$ versus $W_{\dot{F}}$ for $f_{s}=100 \mathrm{Hertz}$ and $R=1.44 \times 10^{-16} \mathrm{~m}^{2}$. Small irregularities are mainly due to numerical errors during the solving of discrete Riccati equation to obtain $K_{\infty}\left(W_{\dot{F}}, f_{s}, R\right)$. with

$$
A_{d}=e^{A T_{s}} \quad B_{d}=\int_{0}^{T_{s}} e^{A t} d t B \quad C_{d}=C
$$

and the measurement $m_{k}^{x}$ is given by Eqs. (33) and (71):

$$
\begin{aligned}
m_{k}^{x} & =x_{k}+v_{k} \\
& =C_{d} X_{k}+v_{k}
\end{aligned}
$$

The global discrete state representing the maglevtube state and the steady-state Kalman filter state is:

$$
\mathcal{X}_{k}=\left[\begin{array}{c}
X_{k} \\
\hat{X}_{k \mid k-1}^{e}
\end{array}\right]
$$

To obtain the associated global state-space model, Eq. (74) is merged in Eqs. (54) and (55):

$$
\begin{aligned}
\hat{X}_{k+1 \mid k}^{e} & =A^{K} \hat{X}_{k \mid k-1}^{e}+B^{K} C_{d} X_{k}+B^{K} v_{k} \\
\hat{F}_{k} & =C^{K} \hat{X}_{k \mid k-1}^{e}+D^{K} C_{d} X_{k}+D^{K} v_{k}
\end{aligned}
$$

and Eqs. (75), (76), (77), (70) leads to the following global representation of the force sensor with $F_{k}^{x}$ and $v_{k}$ as input and $\hat{F}_{k}$ as output:

$$
\begin{aligned}
\mathcal{X}_{k+1} & =A_{g} \mathcal{X}_{k}+B_{g}\left[\begin{array}{c}
F_{k}^{x} \\
v_{k}
\end{array}\right] \\
\hat{F}_{k} & =C_{g} \mathcal{X}_{k}+D_{g}\left[\begin{array}{c}
F_{k}^{x} \\
v_{k}
\end{array}\right]
\end{aligned}
$$

with

$$
\begin{array}{rlr}
A_{g} & =\left[\begin{array}{cc}
A_{d} & 0_{2 \times 3} \\
B^{K} C_{d} & A^{K}
\end{array}\right] & B_{g}=\left[\begin{array}{cc}
B_{d} & 0_{2 \times 1} \\
0_{3 \times 1} & B^{K}
\end{array}\right] \\
C_{g} & =\left[\begin{array}{ll}
D^{K} C_{d} & C^{K}
\end{array}\right] & D_{g}=\left[\begin{array}{ll}
0 & D^{K}
\end{array}\right]
\end{array}
$$

Setting $v_{k}$ to zero in the global state-space model (to study only the impact of $F_{k}^{x}$ input) gives:

$$
\begin{aligned}
\mathcal{X}_{k+1} & =A_{g} \mathcal{X}_{k}+\left[\begin{array}{c}
B_{d} \\
0_{3 \times 1}
\end{array}\right] F_{k}^{x} \\
\hat{F}_{k} & =C_{g} \mathcal{X}_{k}
\end{aligned}
$$

and the global harmonic transfer $H_{g}(j \omega)$ between the force to measure and the force estimated

$$
H_{g}(\omega)=\frac{\hat{F}\left(e^{j \omega}\right)}{F^{x}\left(e^{j \omega}\right)}
$$

is given by:

$$
H_{g}(\omega)=C_{g}\left(e^{j \omega} I_{5 \times 5}-A_{g}\right)^{-1}\left[\begin{array}{c}
B_{d} \\
0_{3 \times 1}
\end{array}\right]
$$

Fig 19 shows the global frequency response of the sensor for different values of $W_{\dot{F}}$ when the calibration is perfectly accurate (matrices $A_{d}$ and $B_{d}$ in Eq. (70) are replaced with the identified matrices given by the calibration stage). 
Sampling frequency $f_{s}$ is set to 1000 Hertz to minimise the effect of the $\mathrm{ZOH}$ in Eq. (70). The step force response (with $v_{k}$ set to zero) is also plotted to see the matching between response time and frequency response of the estimation. Higher is $W_{\dot{F}}$ and larger is the force bandwidth of the sensor. As it is shown, the force bandwidth can be extended beyond the $-3 \mathrm{~dB}$ cutoff frequency of the maglevtube (around $4 \mathrm{~Hz}$ ) and then the phase difference decrease. But in this case the resolution also decreases (see previous Section 5.4). This frequency response can be provided to the end-user to help him adjusting $W_{\dot{F}}$. The assumption at the end of Section 4.1 ruling that $W_{\dot{F}}$ should be set higher for a rapidly varying force than for a slowly varying one is coherent with the way the bandwidth of the sensor is varying with $W_{\dot{F}}$.

Fig. 20 shows the global amplitude frequency response when the calibration is not perfectly accurate. The values chosen are those given in Section 5.2. The scale on Yaxis is linear contrary to Fig. 19(b). Force measurement around the resonant frequency of the maglevtube can lead to non negligible errors.

Fig. 21 shows the five eigen values of $A_{g}$ for $W_{\dot{F}}$ set to different values between $10^{-15} \mathrm{~N}^{2} / \mathrm{Hz}$ and $10^{-18} \mathrm{~N}^{2} / \mathrm{Hz}$. Because of the open-loop design used, the two eigen values very close to the unit circle (inside a small red circle) are the maglevtube poles that are compensated by the zeros associated to the Kalman filter. These two maglevtube poles represent its oscillating and under-damped dynamic with long response time. They do not change if $W_{\dot{F}}$ is modified. The three other poles depend on the value of $W_{\dot{F}}$ and also on the calibration accuracy. Higher is $W_{\dot{F}}$ and closer are the poles to the unit circle right boundary, thus slower is the estimation. The two complex conjugate poles generate an oscillation with a slower dynamic than the real pole that is the fastest pole. The location of the poles does not lead to an instability of the Kalman filter for choice of $W_{\dot{F}}$ even smaller than $10^{-18} \mathrm{~N}^{2} / \mathrm{Hz}$ with double precision floating-point calculation.

\section{Experimental results}

\subsection{Disturbance forces}

The force $\hat{F}_{k}$ is an estimation of $F^{x}(t)$ that represents the sum of all external forces applied on the Maglevtube. Fig. 22 shows $\hat{F}_{k}$ when no external force is artificially applied on the Maglevtube. In this case $\hat{F}_{k}$ is an estimation of all the external disturbance forces applied on the maglevtube along $\vec{x}$ by the environmental noise (see Fig. 1). Because of the maglevtube $-3 \mathrm{~dB}$ cutoff frequency, the most predominant disturbance forces are due to the seismic low frequency vibrations of the sensor base (vibration of magnets $M_{2}$ that induces a vibration of the magnetic field) and to air vibration in infrasound frequencies that generates an acoustic force that is mainly applied on the rear deflector.

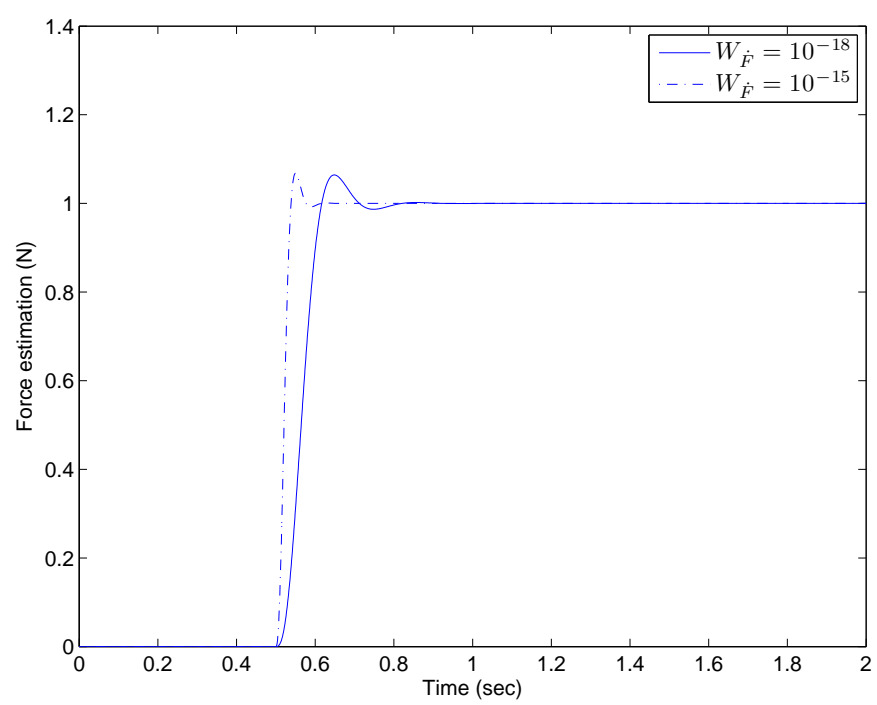

(a) Step force response with noise $v_{k}$ set to zero.

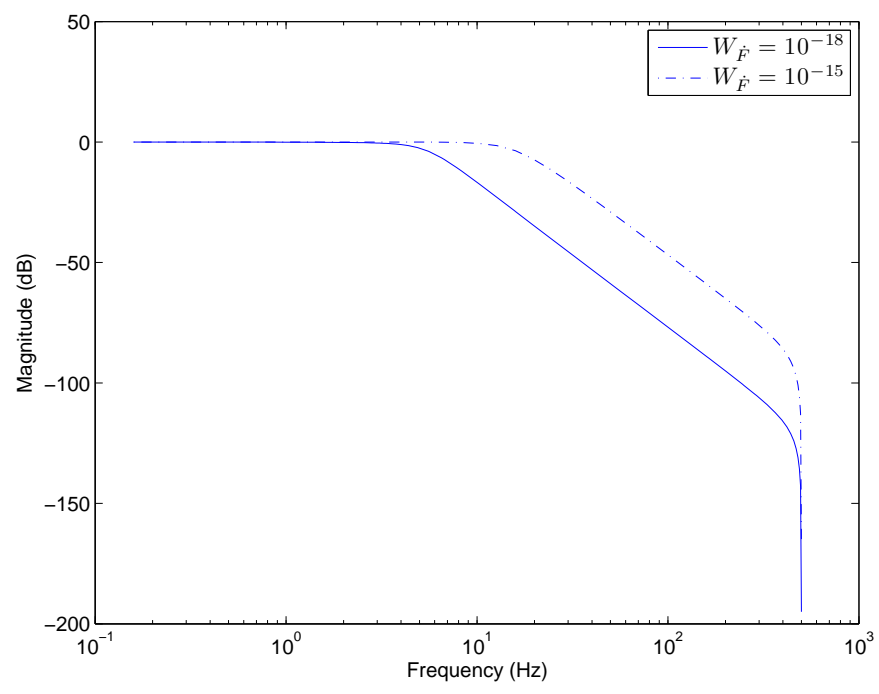

(b) Amplitude of $H_{g}\left(e^{j \omega}\right)$.

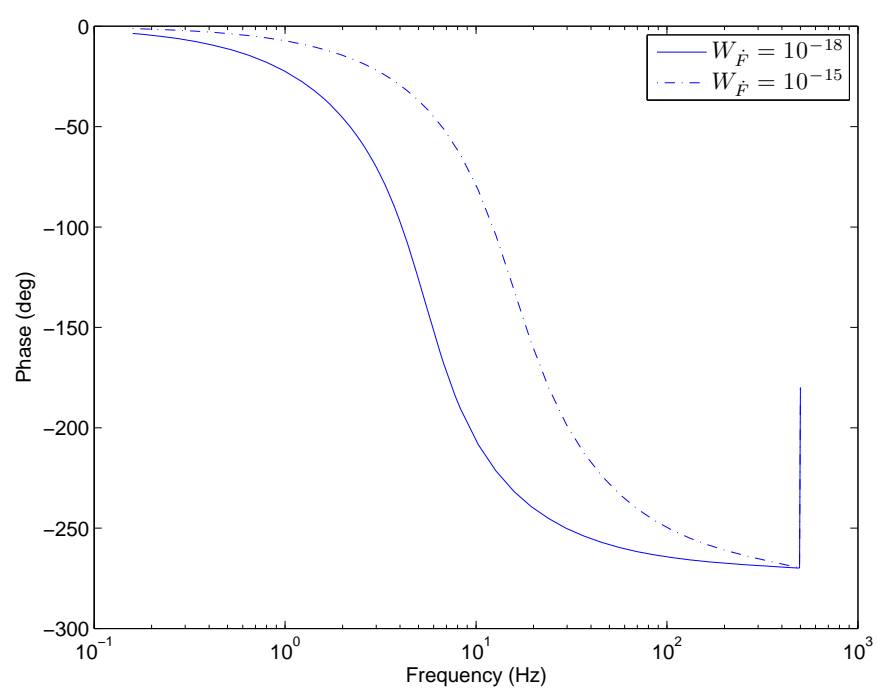

(c) Phase of $H_{g}\left(e^{j \omega}\right)$.

Figure 19: Impact of $W_{\dot{F}}$ on the global frequency response $H_{g}\left(e^{j \omega}\right)$ and the response time of the sensor $\left(f_{s}=1000 \mathrm{Hertz}\right.$ - accurate 13 calibration). 


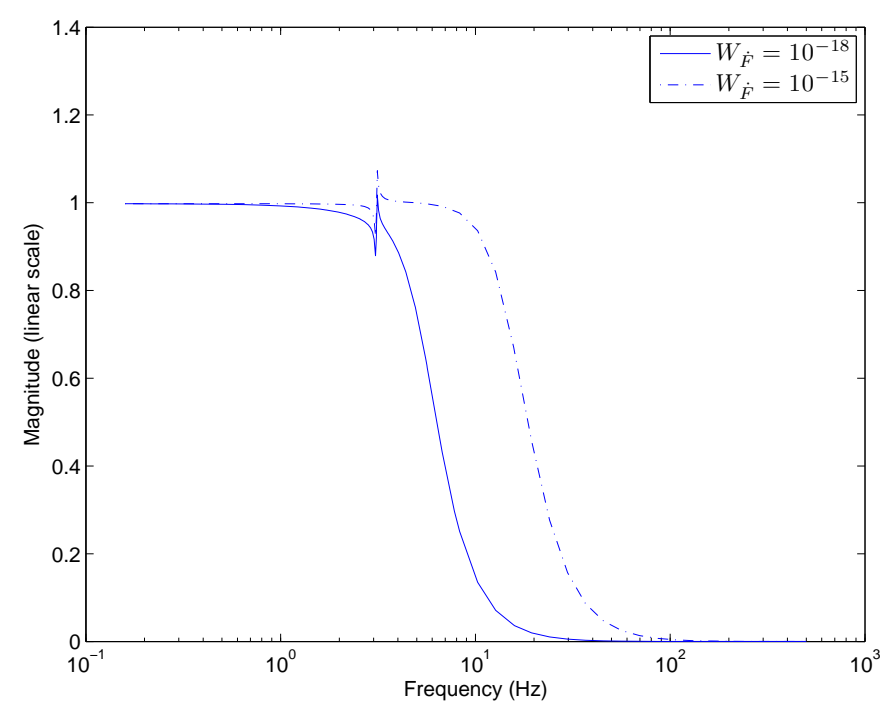

Figure 20: Global amplitude frequency response $H_{g}\left(e^{j \omega}\right)$ with used calibration.

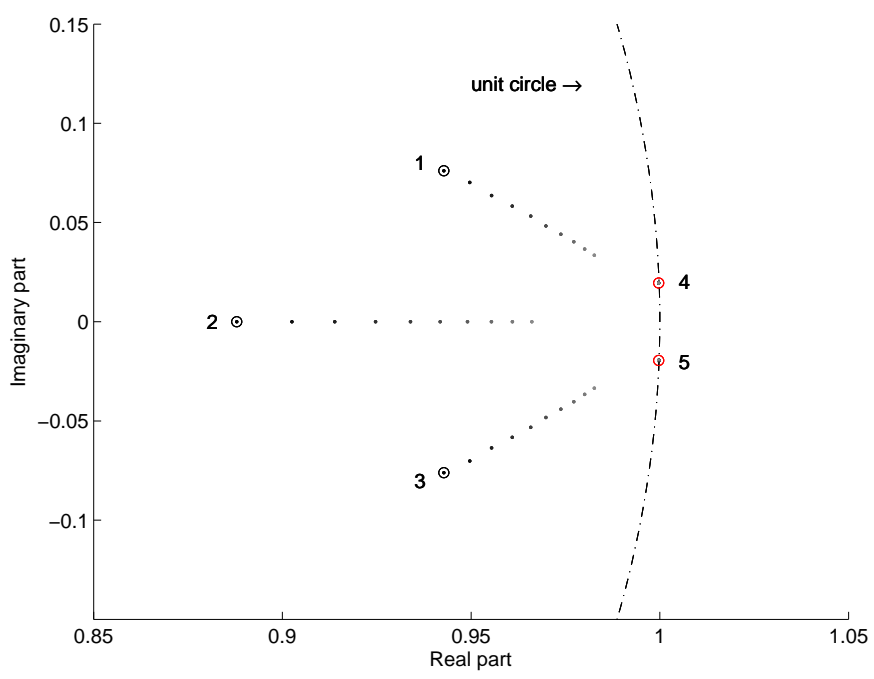

Figure 21: Impact of $W_{\dot{F}}$ on the five eigen values (1 to 5 ) of $A_{g}$. The circled values are associated to $W_{\dot{F}}=10^{-15} \mathrm{~N}^{2} / \mathrm{Hz}$.

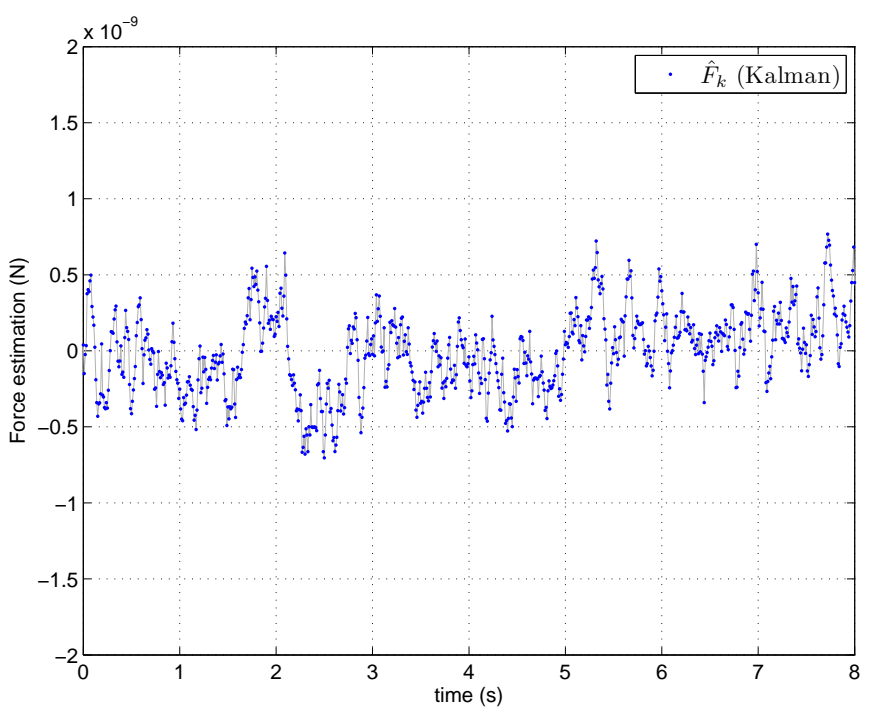

Figure 22: Measurement of the external disturbance force at 2:30 PM.

Extreme care must be taken to reduce as much as possible this environmental noise. With the setup used (sensor enclosed in a chamber put on a antivibration table inside a small room shown in Fig. 23), this disturbance force is probably mainly generated by the antivibration table that insufficiently filters the seismic low frequency vibrations of the massive concrete ground floor. Maximum disturbance force amplitude is inferior to $1 \mathrm{nN}$ and unbiased standard deviation is inferior to $0.3 \mathrm{nN}$.

The presence of vibrations involves that the reference frame $R_{0}$ (attached to the antivibration table) is non Galilean and the fundamental principle of dynamic can not be applied in $R_{0}$ to obtain Eq. (5). Nevertheless, under the hypothesis that the displacement sensor and the magnets $M_{2}$ vibrates in phase (realistic hypothesis in the low frequency domain), it can be shown using a new Galilean reference frame that:

$$
\begin{aligned}
& m \ddot{x}=F^{\prime x}+F_{\text {seismic }}^{x}-K_{m}^{x} x-K_{v}^{x} \dot{x} \\
& m \ddot{x}=F^{x}-K_{m}^{x} x-K_{v}^{x} \dot{x}
\end{aligned}
$$

with

$$
F^{x}=F^{\prime x}+F_{\text {seismic }}^{x}
$$

The force $F_{\text {seismic }}^{x}$ gives the low frequency seismic excitation of the maglevtube that depends on the sensor base low frequency vibration. The force $F^{\prime x}$ represents all the external forces applied on the maglevtube except the seismic excitation. Thus Eq. (87) is the same than Eq. (5). The demonstration of this point and the taking into account of the seismic vibrations (measurement, compensation) is outside the scope of this paper.

\subsection{Pull-off force measurement}

Fig. 25 shows the evolution of the force $\hat{F}_{k}$ during a pull-off force measurement. A planar material is pushed 


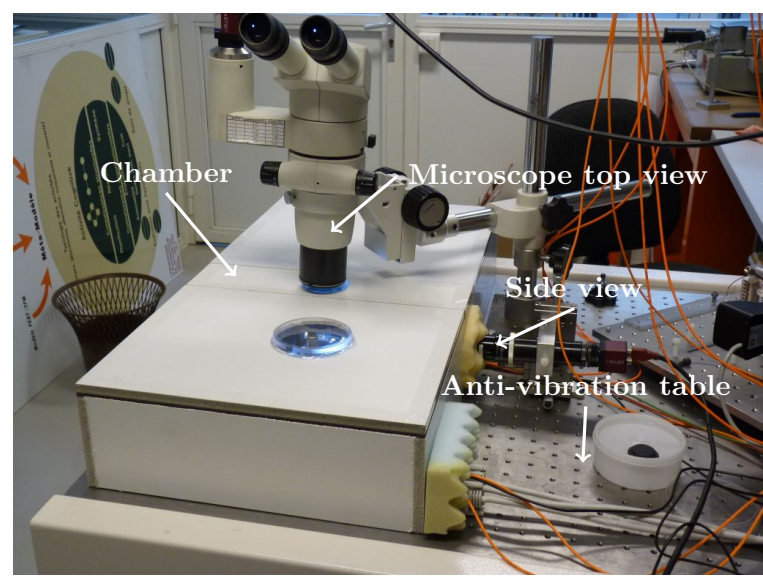

Figure 23: force sensor enclosed in a chamber on a antivibration table.

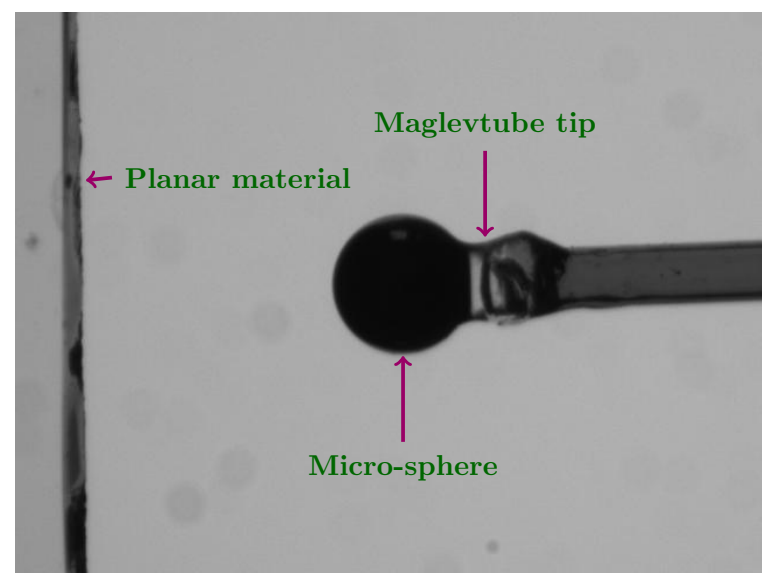

Figure 24: Micro-sphere stuck at the maglevtube tip.

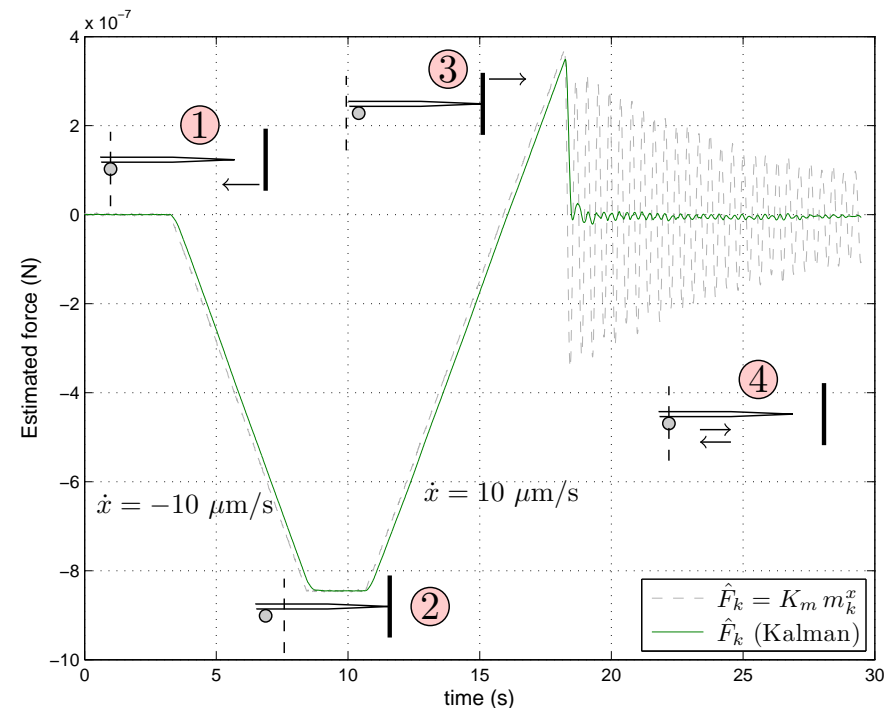

Figure 25: Experimental pull-off force measurement.

against a micro-sphere (see Fig. 24) stuck at the maglevtube tip (loading stage) and then moved back (unloading stage) until the contact is broken between the material and the tip. After this contact loss, the seismic mass is oscillating because of its big inertia but the unknown external force applied on the maglevtube becomes known: it is equal to zero and thus it can be compared with the force estimated with Eq. (3) or Eq. (45). Eq. (3) gives a bad estimation because it is proportional to the displacement of the ZIR. Kalman estimation associated to Eq. (45) gives a better result with a shorter and smaller oscillating transient response. The residual oscillating behaviour can be the result of several causes:

- the identification inaccuracy for the viscous damping coefficient $K_{v}^{x}$,

- the push is not perfectly done in the horizontal direction thus the maglevtube is not aligned with the $x$ axis when the contact is broken. The orientation of the maglevtube around $y$ axis at this instant creates a complex behaviour in the plane $x o z$ that is not taken into account in the one DOF model developed in Section 3.1, thus the Kalman filter does not react correctly,

- the horizontal and vertical seismic excitations of the maglevtube that also create a complex behaviour in the plane $x o z$ defined in Fig. 3 (see previous example).

Fig. 26 also shows a pull-off force measurement but with a non-contact attractive force also applied on the maglevtube sphere before the loading, during the loading and after the contact break. The sphere used is an electrically charged macroscopic sphere (one millimetre diameter, Silicon nitride $\mathrm{Si}_{3} N_{4}$ ). The planar material is a 


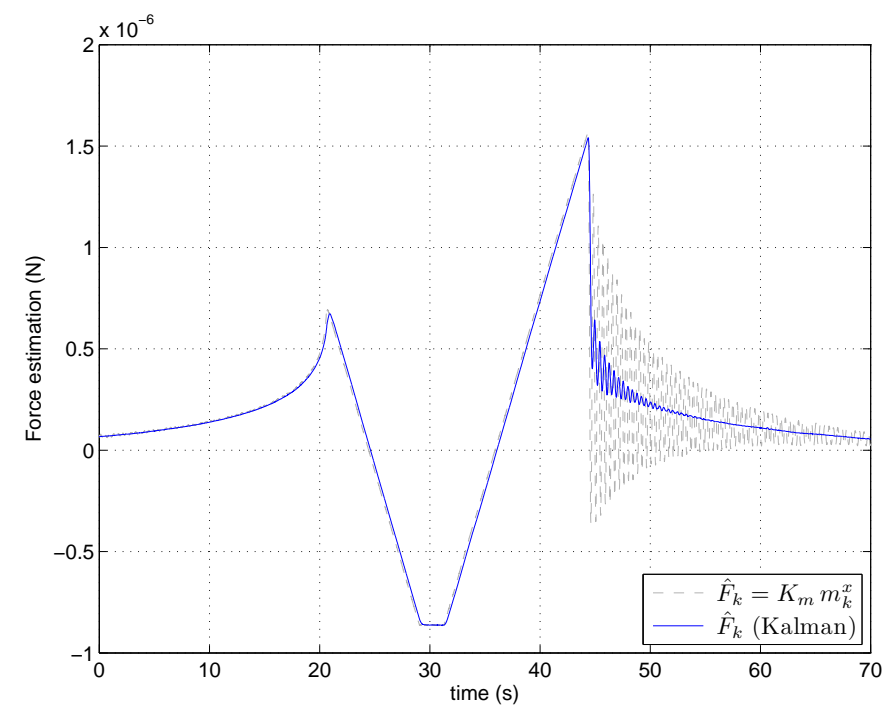

Figure 26: Experimental pull-off force measurement with noncontact attractive force also applied on the Maglevtube.

cover glass (insulating material), thus the most predominant non-contact force is probably an electrostatic force because atmosphere relative humidity was low (no meniscus was observed during the pull-off measurement). The oscillations after the contact break represent the evolution of the non-contact force applied on the sphere when the cover glass is moved back. As the sphere moves towards and backwards the cover glass (because of the maglevtube inertia) the non-contact force is oscillating. The mean of these oscillations has the same mirroring shape than the non-contact force before the loading. Because the speed of the planar material is opposite in both cases, this probably means that the distribution of charges in both material remained the same during all the force measurement.

\section{Conclusion}

Despite the inertia of their mass that should be a great handicap for time-varying force measurement, nanoforce sensors based on a rigid macroscopic seismic mass are not disqualified compared to classical designs based on microscopic elastic cantilevers like AFMs. The design proposed in this paper is based on diamagnetic levitation and makes possible the adjustment of the stiffness of the magnetic springs. Stiffness as low as $0.005 \mathrm{~N} / \mathrm{m}$ can be reached. The linearity of the dynamic behaviour is good for millimetric displacements of the seismic mass, which makes possible the measurement of a large range of force with the same seismic mass. Calibration is quite easy to perform because the mass can be measured with a precision balance. Nevertheless, this design imposes some constraints like the limited bandwidth and the deconvolution of the mass displacement to take care of the behaviour imposed by the mass inertia. This deconvolution is done with a discrete Kalman filter that is using a discretized Wiener process to model the unknown input force uncertainty. This processing requires the adjustment of a single parameter $W_{\dot{F}}$ which directly adjusts a trade-off between the resolution (standard deviation) of $\hat{F}_{k}$ and the bandwidth of the force sensor. This parameter can be modified at any time by the end-user in accordance with its own knowledge on the force to measure. The resolution and frequency response of the sensor can also be plotted to make this choice easier. This deconvolution method is computationally cheap (third-order IIR filter if a steady-state Kalman filter is chosen) and can be implemented in cheap DSP or microcontrollers. Response time shorter than 0.1 second can be reached with a correct $\mathrm{S} / \mathrm{N}$ ratio for forces in the micronewton scale despite the very long settling time of the transducer (20 seconds) and its under-damping. Compared to a simple low-pass filter added on the displacement measurement that will inevitably restrict the bandwidth, the force bandwidth can be extended reasonably four times higher than the displacement bandwidth. The main drawback of this open-loop design is its sensitivity to unwanted external disturbant forces like whose generated by the vibrations of the magnetic field and the vibration of the air in infrasound frequencies. Extreme care must be taken to reduce as much as possible this environmental noise thanks to an antivibration table and a closed chamber. As an outlook, a closed-loop design should be a alternative way to take into account these disturbances in order to cancel them.

\section{Acknowledgment}

This work was supported by the french National Research Agency under STIL $\mu$ FORCE ANR-07-ROBO-0005 contract.

\section{Glossary of symbols}

Order of appearance of mathematic symbols is the same than in the paper.

$K_{m}^{x} \quad$ magnetic stiffness of the force sensor

$K_{v}^{x} \quad$ viscous damping coefficient of the maglevtube

$m$ maglevtube mass

$x(t)$ maglevtube displacement

$F^{x}(t)$ real external force applied to the maglevtube tip

$F(t)$ modelled external force applied to the maglevtube tip

$F_{k} \quad$ discrete modelled external force applied to the maglevtube tip

$\omega(t)$ zero-mean white Gaussian stochastic process used to model uncertainty on $\dot{F}(t)$ 
$W_{\dot{F}} \quad$ power spectral density of stochastic process $\omega(t)$

$X(t)$ continuous state of the maglevtube

$X^{e}(t)$ extended continuous state that is including $F(t)$

$x_{k} \quad$ maglevtube discrete displacement if the external force (real or modelled) is discretized with a zero-order hold

$X_{k} \quad$ discrete state of the maglevtube

$X_{k}^{e} \quad$ extended discrete state including $F_{k}$

$\Omega_{k} \quad$ process noise associated to $X_{k}^{e}$ dynamic

$Q \quad$ covariance matrix of process noise $\Omega_{k}$

$T_{s} \quad$ sampling period used in the force sensor

$f_{s} \quad$ sampling frequency used in the force sensor

$v_{k} \quad$ scalar zero-mean white Gaussian measurement noise

$R \quad$ variance of noise $v_{k}$

$m_{k}^{x} \quad$ noisy measurement of maglevtube displacement

$\hat{X}_{k \mid k-1}^{e}$ prediction of $X_{k}^{e}$ by the Kalman filter

$\hat{X}_{k}^{e} \quad$ estimation of $X_{k}^{e}$ by the Kalman filter

$M_{k} \quad$ set of measurements done between times $t_{1}$ and $t_{k}$ included

$P_{k \mid k-1}$ covariance matrix of prediction error $X_{k}^{e}-\hat{X}_{k \mid k-1}^{e}$ conditioned by measurements done before $t_{k}$

$P_{k} \quad$ covariance matrix of estimation error $X_{k}^{e}-\hat{X}_{k}^{e}$ conditioned by measurements done until $t_{k}$

$K_{k} \quad$ time-varying Kalman gain

$K_{\infty}$ steady-state Kalman gain that depends on $W_{\dot{F}}, f_{s}$ and $R$

$\hat{F}_{k} \quad$ estimated force by the Kalman filter

$\hat{X}_{0}^{e} \quad$ initial a priori estimation of $X_{k}^{e}$

$P_{0} \quad$ initial a priori covariance matrix of estimation error $X_{0}^{e}-\hat{X}_{0}^{e}$

$\omega_{r}(t)$ zero-mean white Gaussian stochastic process used to generate simulated derivative $\dot{F}^{x}(t)$

$W \quad$ power spectral density of $\omega_{r}(t)$

$H^{K} \quad$ SISO transfer function ( $z$-domain) of the steady-state Kalman filter with $m_{k}^{x}$ as input and $\hat{F}_{k}$ as output

$m_{k} \quad$ mean of $\hat{X}_{k \mid k-1}^{e}$ when the steady-state Kalman filter is only excitated by noise $v_{k}$

$S_{k} \quad$ covariance matrix of $\hat{X}_{k \mid k-1}^{e}$ when the steady-state Kalman filter is only excited by noise $v_{k}$ $\mu_{k} \quad$ mean of output noise $n_{k}$ present in $\hat{F}_{k}$

$\Sigma_{k} \quad$ covariance of output noise $n_{k}$ present in $\hat{F}_{k}$

$F_{k}^{x} \quad F^{x}(t)$ applied to a zero-order hold and sampled with frequency $f_{s}$

$\mathcal{X}_{k} \quad$ global state of the force sensor with $F_{k}^{x}$ and $v_{k}$ as input and $\hat{F}_{k}$ as output

$H_{g} \quad$ SISO transfer function ( $z$-domain) of the force sensor with $F_{k}^{x}$ as input and $\hat{F}_{k}$ as output

System, control, output and feed-forward matrices associated to state and output dynamics:

$A, B, C \quad$ modelling of $X(t)$ and $x(t)$ dynamic

$\mathcal{A}, \mathcal{M}, \mathcal{C} \quad$ modelling of $X^{e}(t)$ and $x(t)$ dynamic

$\mathcal{F}, \mathcal{C} \quad$ modelling of $X_{k}^{e}$ and $m_{k}^{x}$ dynamic

$\mathcal{C}_{F} \quad$ output matrix giving $\hat{F}_{k}$ from $\hat{X}_{k}^{e}$

$A^{K}, B^{K}, C^{K}, D^{K}$ modelling of $\hat{X}_{k \mid k-1}^{e}$ and $\hat{F}_{k}$ dynamic

$A_{d}, B_{d}, C_{d} \quad$ modelling of $X_{k}$ and $x_{k}$ dynamic

$A_{g}, B_{g}, C_{g}, D_{g} \quad$ modelling of $\mathcal{X}_{k}$ and $\hat{F}_{k}$ dynamic

\section{References}

[1] M. Sepaniak, P. Datskos, N. Lavrik, and C. Tipple, "Microcantilever transducers: a new approach in sensor technology," Analytical Chemistry, pp. 568-575, November 2002.

[2] P. Rougeot, S. Régnier, and N. Chaillet, "Forces analysis for micro-manipulation," in Proc. of the IEEE International Symposium on Computational Intelligence in Robotics and Automation, espoo, Finland, june 2005, pp. 105-110.

[3] N. Kato, I. Suzuki, H. Kikuta, and K. Iwata, "Force-balancing microforce sensor with an optical-fiber interferometer," $R e$ view of Scientific Instrumentscientific instruments, vol. 68, pp. 2475-2478, June 1997.

[4] F. Arai, T. Sugiyama, T. Fukuda, H. Iwata, and K. Itoigawa, "Micro tri-axial force sensor for 3d bio-micromanipulation," in Proc. of the IEEE International Conference on Robotics and Automation (ICRA), vol. 4, 1999, pp. 2744-2749.

[5] F. Beyeler, S. Muntwyler, and B. J. Nelson, "A six-axis mems force-torque sensor with micro-newton and nano-newtonmeter resolution," Journal of Microelectromechanical Systems, vol. 18, no. 2, pp. 433-441, 2009.

[6] Y. Shen, N. Xi, and W. J. li, "Contact and force control in microassembly," in Proc. of the IEEE International Symposium on Assembly and Task Planning (ISATP), 2003, pp. 60-65.

[7] Y. Fujii, "Methods for generating and measuring the micronewton level forces," Mechanical Systems and Signal Processing, vol. 20, pp. 1362-1371, 2006.

[8] A. Cherry, E. Piat, and J. Abadie, "Analysis of a passive microforce sensor based on magnetic springs and upthrust buoyancy," Sensors and Actuators: A. Physical, vol. 169, pp. 27-36, 2011.

[9] A. Cherry, J. Abadie, and E. Piat, "Modelling and optimization of a floating triangular platform used for nano and microforces sensing," in Proc. of the IEEE/RSJ International Conference on Intelligent Robots and Systems (IROS), 2007, pp. 1118-1123. 
[10] M. Boukallel, J. Abadie, and E. Piat, "Levitated micro-nano force sensor using diamagnetic levitation," in Proc. of the IEEE International Conference of Robotics and Automation (ICRA), september 2003, pp. 3219-3224.

[11] M. Boukallel, E. Piat, and J. Abadie, "Passive diamagnetic levitation: theoretical foundations and application to the design of a micro-nano force sensor," in Proc. of the 2003 IEEE/RSJ international Conference on Intelligent Robots and Systems (IROS), October 2003, pp. 1062-1067.

[12] J. Abadie, E. Piat, S. Oster, and M. Boukallel, "Modeling and experimentation of a passive low frequency nanoforce sensor based on diamagnetic levitation," Sensors and Actuators: A. Physical, vol. 173, pp. 227-237, 2012.

[13] E. Piat, J. Abadie, and S. Oster, "Nanoforce estimation with kalman filtering applied to a force sensor based on diamagnetic levitation," in Proc. of the IEEE International Conference on Intelligent Robots and Systems (IROS), San Francisco, Sept. 25-30 2011, pp. 39-44.

[14] J. R. Pratt, D. T. Smith, D. B. Newell, J. A. Kramar, and E. Whitenton, "Progress toward système international d'unités traceable force metrology for nanomechanics," Journal of Materials Research, vol. 19, pp. 366-379, Jan 2004.

[15] Y. Fujii and H. Fujimoto, "Proposal for an impulse response evaluation method for force transducers," Measurement Science and Technology, vol. 10 (4), pp. N31-N33, 1999.

[16] Y. Fujii, "Proposal for a step response evaluation method for force transducers," Measurement Science and Technology, vol. 14 (10), pp. 1741-1746, 2003.

[17] R. Kumme, "Investigation of the comparison methods for the dynamic calibration of force transducers," Measurement, vol. 23, pp. 239-245, 1998.

[18] Y. Fujii, "A methode for calibrating force transducers against oscillation force," Measurement Science and Technology, vol. 14 (8), pp. 1253-1264, 2003.

[19] G. Demoment, "Image reconstrution and restoration: overview of common estimation structures and problems," IEEE transactions on Acoustics, Speech, and Signal Processing, vol. 37(12), pp. 2024-2036, 1989 .

[20] M. Rakotondrabe and P. Lutz, "Force estimation in a piezoelectric cantilever using the inverse-dynamics-based uio technique," in Proc. of the IEEE International Conference on Robotics and Automation (ICRA), 2009, pp. 2205-2210.

[21] P. S. Maybeck, Stochastic models, estimation and control. Academic Press, 1979, vol. 1.

[22] C. V. Loan, "Computing integrals involving the matrix exponential," IEEE Transactions on Automatic Control, vol. 23, pp. 395-404, June 1978. 